\title{
Supporting Information: The Role of Proton Shuttles in the Reversible Activation of Hydrogen via Metal-Ligand Cooperation
}

\author{
Nicholas E. Smith, ${ }^{\mathrm{a}}$ Wesley H. Bernskoetter, ${ }^{\mathrm{b}}$ and Nilay Hazari ${ }^{\mathrm{a}, *}$ \\ aThe Department of Chemistry, Yale University, P. O. Box 208107, New Haven, Connecticut, \\ 06520, USA. \\ ${ }^{\mathrm{b}}$ The Department of Chemistry, The University of Missouri, Columbia, Missouri, 65211, USA. \\ E-mail: nilay.hazari@yale.edu.
}

SI. General Experimental Considerations $\quad S 2$

SII. Screening of Prospective Proton Shuttles $\quad S 3$

SIII. Methods for Determining Rate Constants for Activation of $\mathrm{H}_{2} \quad S 7$

SIV. Empirical Rate Law Determination for Conversion of 1 to 2

SV. Eyring Plots for the Conversion of 1 to 2

SVI. Kinetic Isotope Effect Measurements for the Conversion of 1 to 2

SVII. Van't Hoff Plots for Addition of $\mathrm{H}_{2}$ to 1

SVIII. Information on Hydrogen Bonding Between 2 and Reaction Additives S26

SIX. Infrared Spectroscopic Measurements of Complex 2 in the Presence of ${ }^{t} \mathrm{BuOH} \quad S 27$

SX. Empirical Rate Law Determinations for Conversion of 3 to 4 and 5 to 6

SXI. Information on Effect of Additives on $\mathrm{H}_{2}$ Activation by 3 and $\mathbf{5} \quad S 32$

$\begin{array}{ll}\text { SXII. References } & \text { S34 }\end{array}$ 


\section{SI. General Experimental Considerations}

Experiments were performed under a dinitrogen atmosphere in an M. Braun glovebox or using standard Schlenk techniques unless otherwise noted. Under typical operating conditions, the glovebox was not purged between uses of benzene, diethyl ether, pentane, THF, or toluene. As a consequence, each solvent should be assumed to contain trace amounts of the others unless otherwise noted. For kinetic experiments, toluene was kept rigorously free of diethyl ether and THF. All moisture and air-sensitive liquids were either transferred inside the glovebox or using a stainless-steel cannula or a gas-tight syringe using a Schlenk line. Solvents were dried by passage through a column of activated alumina and stored under dinitrogen unless otherwise noted. All commercial chemicals were used as received except where noted. ${ }^{\mathrm{iPr}} \mathrm{PN}^{\mathrm{H}} \mathrm{P}$ was purchased from Strem. High-purity research-grade dihydrogen was obtained from Airgas, Inc. The alcohols $p$ methyl-2-phenyl-2-propanol and tert-butanol were purchased from Sigma-Aldrich and sublimed or distilled in vacuo prior to use. The alcohols 2-phenyl-2-propanol and p-bromo-2-phenyl-2propanol were purchased from TCI America, Inc. and sublimed prior to use. Sodium borodeuteride, tert-butanol- $d_{1}$, and deuterated solvents were obtained from Cambridge Isotope Laboratories. $\mathrm{C}_{6} \mathrm{D}_{6}$ and $\mathrm{C}_{6} \mathrm{D}_{5} \mathrm{CD}_{3}$ were dried over sodium metal or by passage through a plug of activated alumina. The complexes $\left.\left[\left({ }^{\mathrm{iPr} P N}{ }^{\mathrm{HP}}\right) \mathrm{Fe}(\mathrm{Cl})_{2}(\mathrm{CO})\right],{ }^{1}\left[{ }^{\mathrm{iPr} P N P}\right) \mathrm{Fe}(\mathrm{H})(\mathrm{CO})\right] \quad(\mathbf{1}),{ }^{2}$ $\left[\left({ }^{\mathrm{iPr} P N P}\right) \mathrm{Ru}(\mathrm{H})(\mathrm{CO})\right](\mathbf{3}),{ }^{3}$ and $\left[\left({ }^{\mathrm{tBu}} \mathrm{PN}{ }^{\mathrm{py}} \mathrm{P}\right) \mathrm{Ru}(\mathrm{H})(\mathrm{CO})\right]^{4}{ }^{(5)}$ were synthesized according to procedures reported previously in the literature. NMR spectra were recorded on Bruker AMX-400 or AMX-500 spectrometers at ambient probe temperatures, unless otherwise noted. Chemical shifts are reported in ppm with respect to residual internal protio solvent for ${ }^{1} \mathrm{H}$ and ${ }^{13} \mathrm{C}\left\{{ }^{1} \mathrm{H}\right\} \mathrm{NMR}$ spectra. ${ }^{31} \mathrm{P}\left\{{ }^{1} \mathrm{H}\right\}$ NMR spectra are referenced via the ${ }^{1} \mathrm{H}$ resonances based on the relative gyromagnetic ratios. ${ }^{5}$ UV-Vis data were collected on a Hewlett-Packard 8453 diode array spectrophotometer. The temperature for UV-Vis experiments was controlled using a Unisoku Unispeks cryostat. Infrared data were obtained on a Bruker ALPHA FTIR spectrometer with a solution transmittance attachment inside an $\mathrm{N}_{2}$-filled glovebox. The observation cell used for solution state infrared data collection was composed of $\mathrm{CaF}$ windows with a path length of 0.2 $\mathrm{mm}$. 


\section{SII. Screening of Prospective Proton Shuttles}

Certain protic molecules, such as methanol, thiols, and water, can undergo 1,2-addition across the Fe-N $\pi$-bond of 1 (Scheme S1). ${ }^{6}$ Some of these molecules, primarily non-tertiary alcohols, can also undergo dehydrogenation to form $\mathbf{2}$ directly from $\mathbf{1}$ and the additive, even at room temperature. For example, methanol can be dehydrogenated by $\mathbf{1}$ to form $\mathbf{2}$ and formaldehyde. ${ }^{6}$ Representative spectra of the 1,2-addition of a small molecule to $\mathbf{1}$ are provided below (Figure S1).

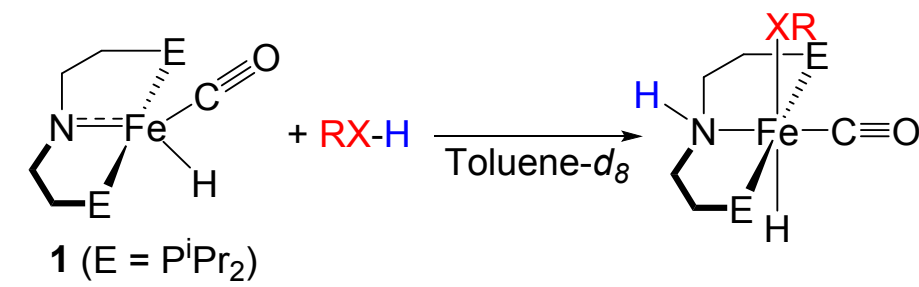

Where $\mathrm{RX}-\mathrm{H}$ is a protic moiety such as those present in: alcohols, thiols, terminal alkynes, water, and ureas

Scheme S1: General depiction of a 1.2-addition reaction across the Fe-N $\pi$-bond of $\mathbf{1}$.

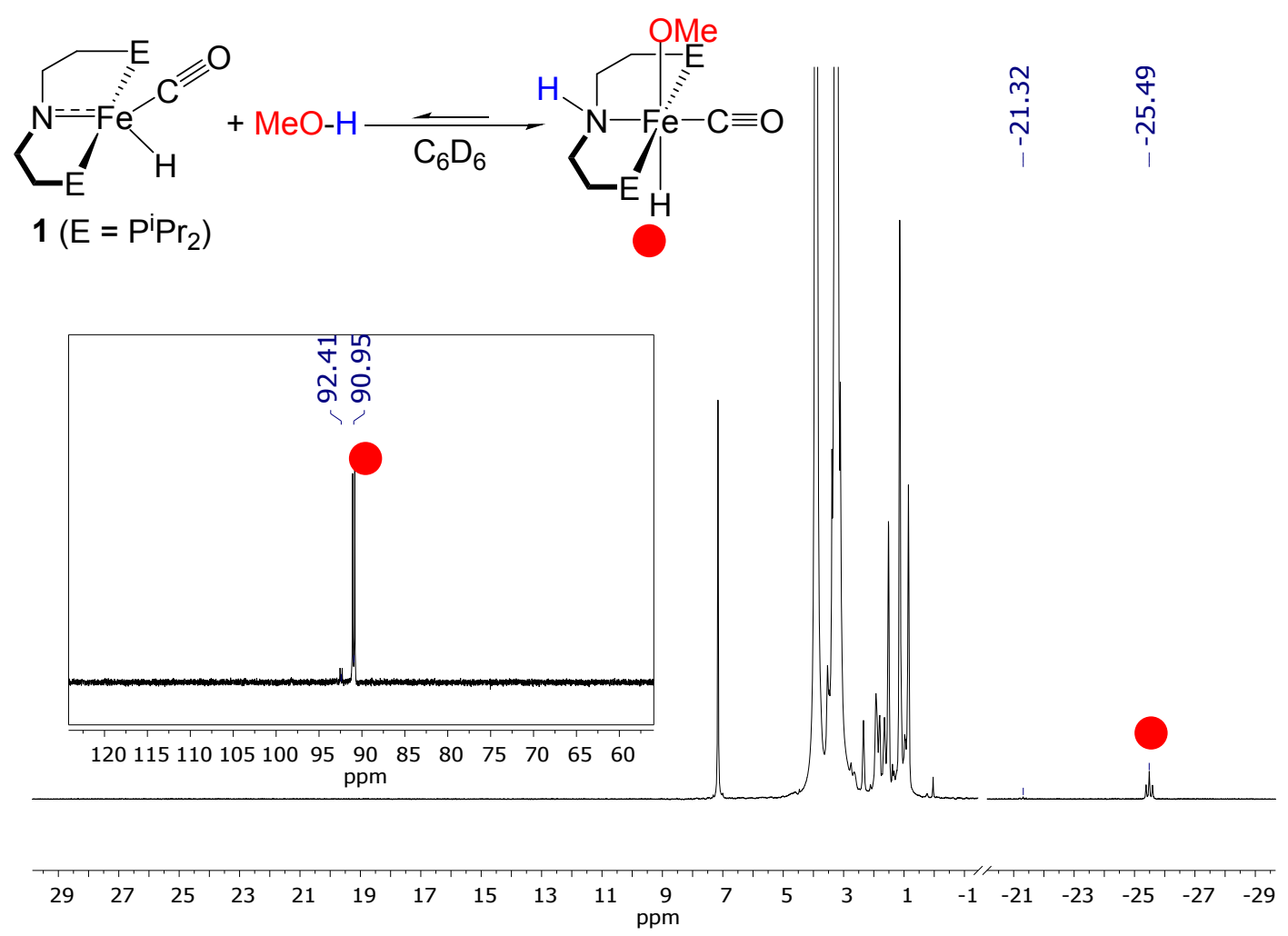

Figure S1: ${ }^{1} \mathrm{H}$ NMR spectrum ( ${ }^{31} \mathrm{P}$ NMR spectrum inset) of the reaction between 1 and 100 equiv. of MeOH, which results in 1,2-addition across the Fe-N $\pi$-bond of 1 . The ${ }^{31} \mathrm{P}$ NMR spectrum shows a second product of unknown identity. At lower equiv. of $\mathrm{MeOH}$ (5-10 equiv.), the dihydride complex 2 is also visible due to competitive dehydrogenation of $\mathrm{MeOH}$ to form formaldehyde.

A variety of small molecules were tested as prospective proton shuttles (Table S1) using the procedure described below. Initially, ${ }^{1} \mathrm{H}$ and ${ }^{31} \mathrm{P}$ NMR spectroscopy was used to determine if $\mathbf{1}$ underwent a side reaction with the prospective shuttle. Kinetic traces for the conversion of $\mathbf{1}$ and 
$\mathrm{H}_{2}$ to 2 in the presence of small molecules were then recorded for those additives that did not exhibit side-reactivity with $\mathbf{1}$ to determine if they acted as proton shuttles. The results are described in Table S1.

Representative procedure for determining if background reactions occurred between 1 and prospective proton shuttles: In a glovebox, a stock solution of $\mathbf{1}(9.20 \mathrm{mM})$ was prepared in $\mathrm{C}_{6} \mathrm{D}_{6}$, and then an aliquot was transferred into a J-Young NMR tube $(750 \mu \mathrm{L}, 6.90 \mu \mathrm{mols})$. A sample (95-105 equiv. unless otherwise noted) of the small molecule (e.g. diisopropylamine, 100 $\mu \mathrm{L}, 0.709$ mmols, 103 equiv.) was then added to the NMR tube. The tube was sealed, removed from the glovebox and shaken until the sample was visually homogeneous. After allowing to sit for 5 minutes, ${ }^{1} \mathrm{H}$ and ${ }^{31} \mathrm{P}$ NMR spectra were recorded. Molecules which led to the formation of 2, identified by the diagnostic hydride resonances in the ${ }^{1} \mathrm{H}$ NMR, are labelled as undergoing "dehydrogenation". For molecules labelled as undergoing 1,2-addition, this was signified by both a shift in the ${ }^{31} \mathrm{P}$ NMR resonance of the ligand as well as a shift in the hydride resonance of $\mathbf{1}$ in the ${ }^{1} \mathrm{H}$ NMR spectrum. The chemical shift changes were similar to that observed previously for 1,2 -addition to $\mathbf{1}$, however, none of these complexes were isolated or fully characterized. ${ }^{6}$ 


\begin{tabular}{|c|c|c|c|}
\hline Small Molecule & $\begin{array}{c}\text { Side- } \\
\text { reactivity } \\
\text { with } \mathbf{1} \text { ? }\end{array}$ & $\begin{array}{l}\text { If applicable, what was side- } \\
\text { reactivity? }\end{array}$ & $\begin{array}{c}\text { Active as a } \\
\text { proton } \\
\text { shuttle? }\end{array}$ \\
\hline${ }^{\mathrm{t}} \mathrm{BuOH}$ & No & - & Yes \\
\hline 2-phenyl-2-propanol (H-Ar) & No & - & Yes \\
\hline $\begin{array}{l}\text { p-bromo-2-phenyl-2-propanol } \\
\text { (Br-Ar) }\end{array}$ & No & - & Yes \\
\hline $\begin{array}{c}\text { p-methyl-2-phenyl-2-propanol } \\
\text { (Me-Ar) }\end{array}$ & No & - & Yes \\
\hline $\begin{array}{l}p \text {-trifluoromethyl-2-phenyl-2- } \\
\text { propanol }\end{array}$ & Yes & 1,2-addition & Yes* \\
\hline Methanol & Yes & Dehydrogenation, 1,2-addition & - \\
\hline Ethanol & Yes & Dehydrogenation, 1,2-addition & - \\
\hline Isopropanol & Yes & Dehydrogenation, 1,2-addition & - \\
\hline Diphenylmethanol & Yes & Dehydrogenation, 1,2-addition & - \\
\hline Phenol & Yes & 1,2-addition & - \\
\hline 2,6-dimethylphenol & Yes & 1,2-addition & - \\
\hline${ }^{\mathrm{t}} \mathrm{BuNH}_{2}$ & No & - & No \\
\hline${ }^{\mathrm{t} B u S H}$ & Yes & 1,2-addition & - \\
\hline Aniline & No & - & No \\
\hline Diphenylamine & No & - & No \\
\hline TBD & Yes & 1,2-addition & - \\
\hline Diisopropylamine & No & - & No \\
\hline Hexamethyldisilazane & No & - & No \\
\hline Morpholine & No & - & No \\
\hline Piperidine & No & - & No \\
\hline 1,2,3,4-tetrahydroquinoline & No & - & No \\
\hline Adamantylamine & No & - & No \\
\hline Benzylamine & No & - & No \\
\hline Formanilide & Yes & 1,2-addition & - \\
\hline Phenylacetylene & Yes & 1,2-addition & - \\
\hline Urea & Yes & 1,2-addition & - \\
\hline Water & Yes & 1,2-addition & Yes** \\
\hline
\end{tabular}

Table S1: Protic species tested for compatibility with $\mathbf{1}$ and their performance as proton shuttles (if applicable). *While a rate enhancement was observed for the addition of $\mathrm{H}_{2}$ to $\mathbf{1}$, rate constants could not be reliably determined because the product 2 was in equilibrium with the direct 1,2addition product from the reaction of $p$-trifluoromethyl-2-phenyl-2-propanol with 1 . ${ }^{* *}$ While a rate enhancement is observed for the addition of $\mathrm{H}_{2}$ to 1 , rate constants could not be reliably determined because the starting material $\mathbf{1}$ is in equilibrium with the 1,2-addition product of water (see Figure S2). 


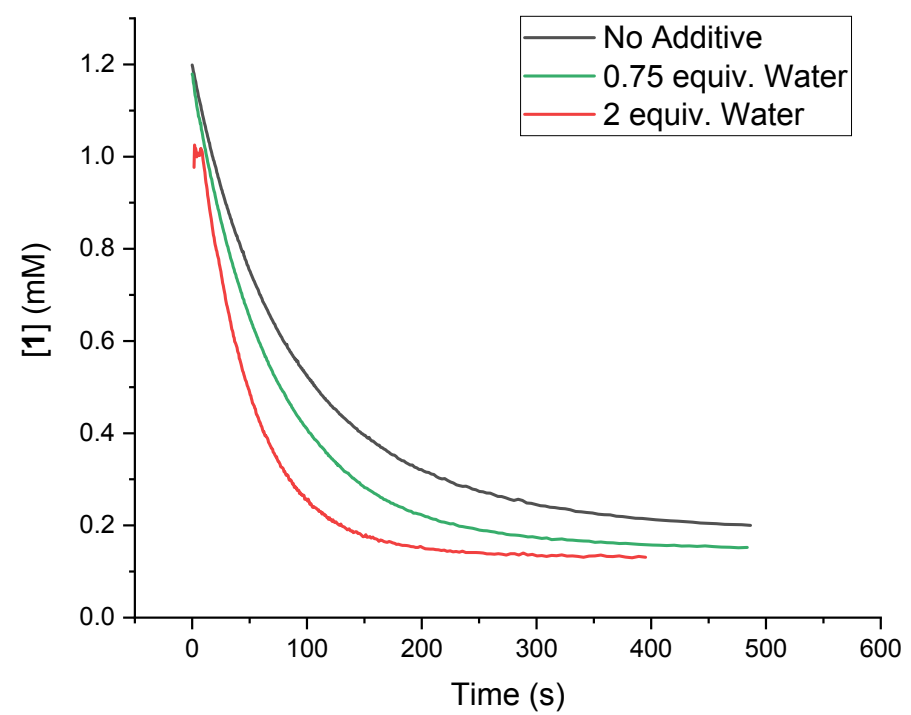

Figure S2: Line plot of kinetic traces for the conversion of $\mathbf{1}$ and $\mathrm{H}_{2}$ to 2 in the presence of 0.75 equiv. (green) and 2 equiv. (red) of water. 
SIII. Methods for Determining Rate Constants for Activation of $\mathrm{H}_{2}$

Data Collection: All kinetic measurements were made using a Hewlett-Packard 8453 diode array spectrophotometer with temperatures controlled using a Unisoku Unispeks cryostat. In a typical experiment, a reaction-type cuvette, as previously described and pictured below, was used to prepare the sample (Figure S2). ${ }^{7}$ All three starting transition metal complexes, 1, 3, and 5, have been reported to undergo the 1,2-addition of $\mathrm{H}_{2}$ upon the introduction of $1 \mathrm{~atm}$ of $\mathrm{H}_{2}$ at $25{ }^{\circ} \mathrm{C}$. . $^{-}$ $3,4 b, 8$ Control reactions in a J-Young NMR tube were performed where a solution of $\mathbf{1}, \mathbf{3}$, or $\mathbf{5}$ was subjected to three freeze-pump-thaw cycles followed by addition of 1 atm of $\mathrm{H}_{2}$ from a Schlenk manifold, and the products observed by ${ }^{1} \mathrm{H}$ and ${ }^{31} \mathrm{P}$ NMR matched spectra reported in the literature. ${ }^{2,4 b, 9}$

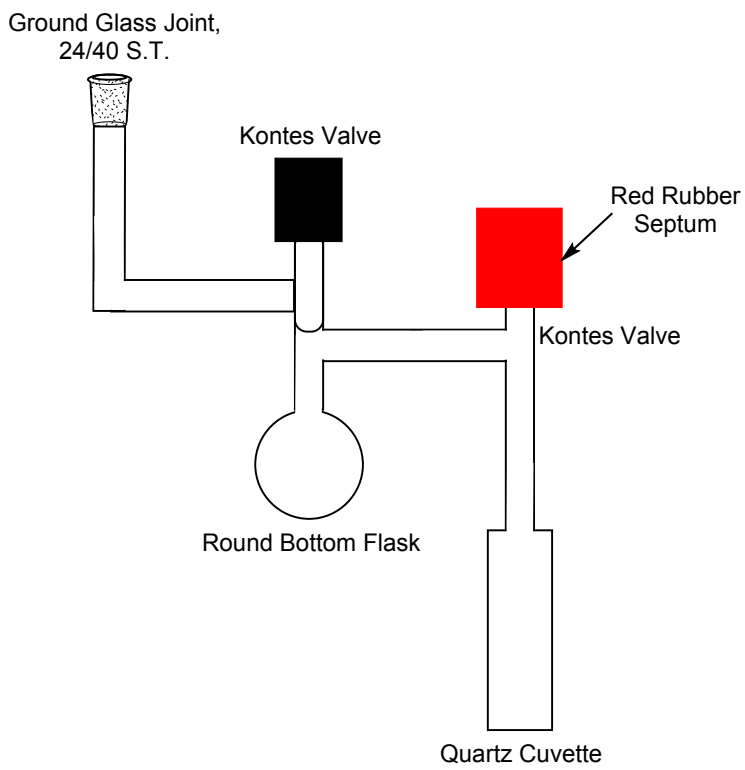

Figure S3: Schematic of reaction cuvette design, made of borosilicate glass with a quartz observation cuvette, for kinetic experiments.

Example procedure for kinetic monitoring of a reaction between 1 and $\mathbf{H}_{2}$ : In a glovebox, a stock solution of 1 was prepared in toluene $(40.0 \mathrm{mM})$. This was loaded into a Hamilton SampleLock syringe (for a typical trial, $0.075 \mathrm{~mL}$ of stock solution, $3.0 \mu$ mols 1 ) which was sealed using the locking valve. A second stock solution containing the desired additive $(40.0 \mathrm{mM}$ in toluene) was prepared. $0.750 \mathrm{~mL}$ of this stock solution was transferred into the round-bottom flask of the reaction vessel (Figure S2) using a graduated syringe. For example, for an experiment with 10 equiv. of ${ }^{t} \mathrm{BuOH}, 30 \mu$ mols of ${ }^{t} \mathrm{BuOH}$ were added to the reaction flask. (Note: if an experiment was performed in the absence of an additive, this step was not performed.) The solution in the round bottom of the reaction vessel was then diluted with toluene until the total volume of the solution in the vessel was $2.925 \mathrm{~mL}$, as measured by a graduated syringe. The Kontes valve directly above the cuvette in the reaction vessel was sealed with a red rubber septum lined with Teflon and a Kontes valve pin was used to seal the reaction vessel above the round bottom flask. Using the ground glass joint, the reaction vessel was attached to a Schlenk manifold. Then, the solution in the round bottom was subjected to three freeze-pump-thaw cycles to remove the nitrogen atmosphere. At this point, the reaction vessel was refilled with $\mathrm{H}_{2}$ from the Schlenk manifold and the pressure adjusted to the appropriate pressure for the desired experiment. The reaction vessel was then resealed and the solution tipped into the cuvette part of the reaction vessel. The cuvette 
was placed into the UV-Vis spectrometer and the temperature was allowed to equilibrate while stirring ( $c a .5$ minutes). A blank spectrum was then taken of the cuvette to be used as a baseline spectrum during the reaction. Monitoring of the reaction solution by the spectrometer was started, followed by immediate injection of 1 into the cuvette while stirring. This caused the final volume of solution in the cuvette to reach $3.000 \mathrm{~mL}$, which is the volume used to calculate the initial concentration of each of the reagents (1.0 mM 1, $10.0 \mathrm{mM}$ t BuOH for 10 equiv.). The full UV-Vis spectrum from $190 \mathrm{~nm}$ to $1100 \mathrm{~nm}$ was recorded every 0.5 seconds for the first 120 seconds, with further spectra recorded with an increase in delay time of $2 \%$ between each spectrum if applicable.

Example procedure for kinetic monitoring of a reaction between 3 and $\mathrm{H}_{2}$ : In a glovebox, a stock solution of 3 was prepared (10.6 $\mathrm{mM}$ in toluene). This was loaded into a Hamilton SampleLock syringe (for a typical trial, $0.060 \mathrm{~mL}$ of stock solution, $0.64 \mu \mathrm{mols} 3$ ) which was sealed using the locking valve. A second stock solution containing the desired additive $(10.6 \mathrm{mM}$ in toluene) was prepared. $0.060 \mathrm{~mL}$ of this stock solution was transferred into the round-bottom flask of the reaction vessel (Figure S2) using a graduated syringe. For example, for an experiment with 1 equiv. of ${ }^{\text {t }} \mathrm{BuOH}, 0.64 \mu \mathrm{mols}$ of ${ }^{\mathrm{BuOH}}$ were added to the reaction flask. (Note: if an experiment was performed in the absence of an additive, this step was not performed.) The solution in the round bottom flask of the reaction vessel was then diluted with toluene until the total volume of the solution in the vessel was $2.94 \mathrm{~mL}$, as measured by a graduated syringe. The Kontes valve above the cuvette in the reaction vessel was sealed with a red rubber septum lined with Teflon, while a Kontes valve pin was used to seal the reaction vessel above the round bottom flask. Using the ground glass joint, the reaction vessel was attached to a Schlenk manifold. Then, the solution in the round bottom was subjected to three freeze-pump-thaw cycles to remove the nitrogen atmosphere. At this point, the reaction vessel was refilled with $\mathrm{H}_{2}$ from the Schlenk manifold and the pressure adjusted to the appropriate pressure for the desired experiment. The reaction vessel was resealed and the solution tipped into the cuvette part of the reaction vessel. The cuvette was placed into the UV-Vis spectrometer and the temperature was allowed to equilibrate while stirring (ca. 5 minutes). A blank spectrum was taken of the cuvette to be used as a baseline spectrum during the reaction. Monitoring of the reaction solution by the spectrometer was started, followed by immediate injection 3 into the cuvette while stirring. This caused the final volume of solution in the cuvette to reach $3.000 \mathrm{~mL}$, which is the volume used to calculate the initial concentration of

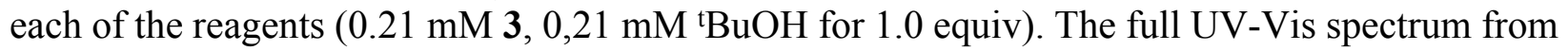
$190 \mathrm{~nm}$ to $1100 \mathrm{~nm}$ was recorded every 0.5 seconds for 100 seconds.

Example procedure for kinetic monitoring of a reaction between 5 and $\mathbf{H}_{2}$ : In a glovebox, a stock solution of $\mathbf{5}$ was prepared $(5.0 \mathrm{mM}$ in toluene). This was loaded into a Hamilton SampleLock syringe (for a typical trial, $0.060 \mathrm{~mL}$ of stock solution, $0.30 \mu$ mols 5 ) which was then sealed at the locking valve. A second stock solution containing the desired additive $(5.0 \mathrm{mM}$ in toluene) was prepared. $0.060 \mathrm{~mL}$ of this stock solution was transferred into the round-bottom flask of the reaction vessel (Figure S2) using a graduated syringe. For example, for an experiment with 1 equiv. of ${ }^{\mathrm{t}} \mathrm{BuOH}, 0.30 \mu \mathrm{mols}$ of ${ }^{\mathrm{t}} \mathrm{BuOH}$ were added to the reaction flask. (Note: if an experiment was performed in the absence of an additive, this step was not performed.) The solution in the round bottom of the reaction vessel was then diluted with toluene until the total volume of the solution in the vessel was $2.94 \mathrm{~mL}$, as measured by a graduated syringe. The Kontes valve above the cuvette was sealed with a red rubber septum lined with Teflon and a Kontes valve pin was used to seal the reaction vessel above the round bottom flask. Using the ground glass joint, the reaction 
vessel was attached to a Schlenk manifold. Then, the solution in the round bottom flask was subjected to three freeze-pump-thaw cycles to remove the nitrogen atmosphere. At this point, the reaction vessel was refilled with $\mathrm{H}_{2}$ from the Schlenk manifold and the pressure adjusted to the appropriate pressure for the desired experiment $(1 \mathrm{~atm})$. The reaction vessel was resealed and the solution tipped into the cuvette part of the reaction vessel. The cuvette was placed into the UV-Vis spectrometer and the temperature was allowed to equilibrate while stirring ( $c a .5$ minutes). A blank spectrum was then taken of the cuvette to be used as a baseline spectrum during the reaction. Monitoring of the reaction solution by the spectrometer was started, followed by immediate injection of the metal complex into the cuvette while stirring. This caused the final volume of solution in the cuvette to reach $3.000 \mathrm{~mL}$, which is the volume used to calculate the initial

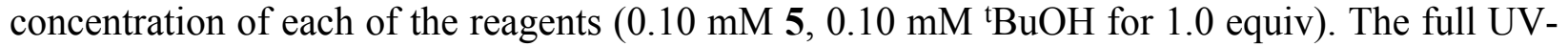
Vis spectrum from $190 \mathrm{~nm}$ to $1100 \mathrm{~nm}$ was recorded every 0.5 seconds for 100 seconds.

Data Workup; Determining Rate Constants:

As the spectrometer was started before the metal complex solution was added, the first 4-6 seconds of data during which no reaction was occurring were removed before modelling. Concentration profiles were obtained from several different wavelengths to ensure self-consistency in the observed rate constants $\left(k_{\text {obs }}\right)$. The concentration of $\mathrm{H}_{2}$ in toluene, was determined from reported literature values. ${ }^{10}$ In reactions using 1 , no induction period or sigmoidal kinetics were observed for the unshuttled reaction, which would indicate autocatalysis by the N-H moiety of the product complex 2 .

Observed rate constants $\left(k_{\mathrm{obs}}\right)$ for the addition of $\mathrm{H}_{2}$ to a given metal complex were obtained using the method of initial rates. Elementary rate constants were obtained using the COPASI kinetics modelling package and concentration profiles for the starting complex. ${ }^{11}$ In order to obtain reliable fits for the conversion of $\mathbf{1}$ to $\mathbf{2}$, COPASI required the input of equilibrium constants, obtained from variable-temperature ${ }^{1} \mathrm{H}$ NMR spectroscopy (vide infra), to determine the forward and reverse rate constants. Alternative models, including the addition of unobserved pre-equilibrium species, did not dramatically improve the quality of the COPASI fit. Representative UV-Vis data for each reaction monitored $(\mathbf{1 \rightarrow 2}, \mathbf{3} \rightarrow \mathbf{4}, \mathbf{5} \rightarrow \mathbf{6})$ are shown below (Figure S3). Arrows show the direction in which absorption changes during the reaction from starting material to the final state (products or equilibrium mixture).

Elementary rate constants were determined from COPASI by modelling concentration profiles of the conversion of the starting amido/dearomatized complexes to the dihydrides. Concentration profiles were obtained from the absorbance of the reaction mixture at a local maximum in the UVVis spectrum of the starting material. The wavelength that was monitored to derive concentration profiles for each reaction is shown in Table S2. All rate constants were determined using this method unless otherwise noted. All reactions in the absence of any additive were modelled using Eq. 1, but the conversions of $\mathbf{3} \rightarrow \mathbf{4}$ and $\mathbf{5} \rightarrow \mathbf{6}$ were modelled as irreversible as no detectable concentration of the starting complex was present as determined by ${ }^{1} \mathrm{H}$ NMR spectroscopy under $1 \mathrm{~atm}$ of $\mathrm{H}_{2}$. The value of $k_{1}$ was allowed to freely refine using a Levenberg-Marquardt leastsquares refinement. For reactions that were irreversible, the reaction was modelled as such. For reactions that were reversible, the value of $k_{-1}$ was fixed to the value of $k_{1}$ using Eq. 2 , where $\mathrm{K}_{\mathrm{eq}}$ is the equilibrium constant for $\mathbf{1}, \mathrm{H}_{2}$, and $\mathbf{2}$ as determined by NMR spectroscopy. Van't Hoff plots 
(See Section SVII) were used to determine the value of $\mathrm{K}_{\mathrm{eq}}$ for all experiments. Representative examples of COPASI output fits for the conversion of $\mathbf{1}$ to $\mathbf{2}$ are in Figures S4 and S5.

(a)

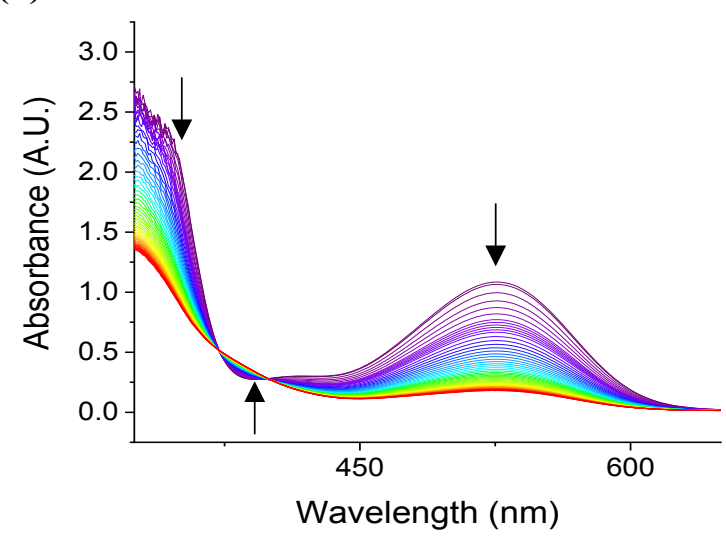

(b)

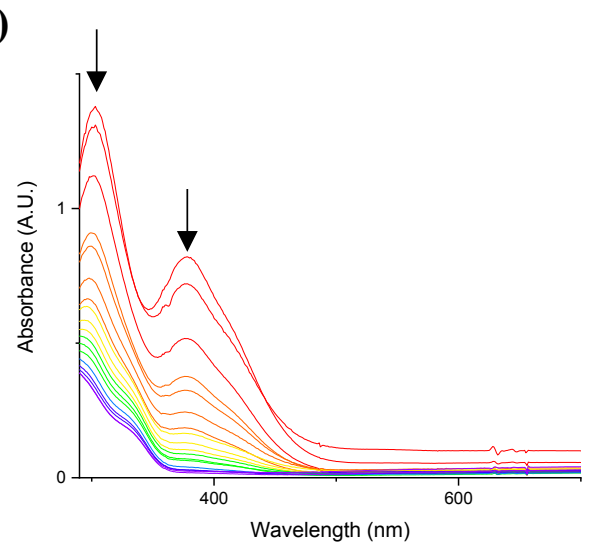

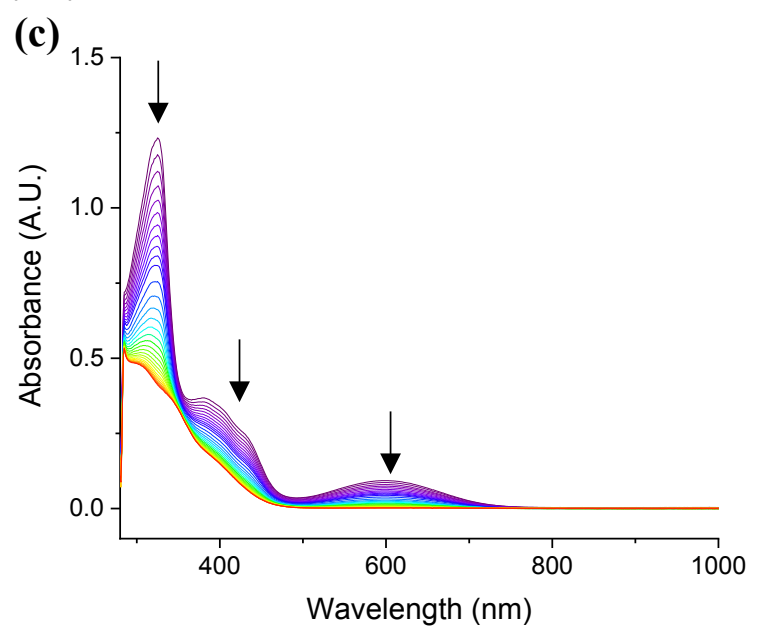

Figure S4: Representative spectra for the conversion of (a) 1 and $\mathrm{H}_{2}$ to 2. Conditions: Toluene solvent, $[\mathbf{1}]_{0}=1.00 \mathrm{mM}, \mathrm{p}\left(\mathrm{H}_{2}\right)=1 \mathrm{~atm}, 4$ equiv. ${ }^{\mathrm{t}} \mathrm{BuOH}\left[{ }^{\mathrm{t}} \mathrm{BuOH}\right]_{0}=40.0 \mathrm{mM}$, spectra recorded every $0.5 \mathrm{~s}$ (shown every $1.0 \mathrm{~s}$ for clarity), for first $120 \mathrm{~s}$, then increment by $2 \%$ for each data point recorded (every $3^{\text {rd }}$ data point shown for clarity), (b) 3 and $\mathrm{H}_{2}$ to 4 . Conditions: Toluene solvent, $[3]_{0}=0.282 \mathrm{mM}, \mathrm{p}\left(\mathrm{H}_{2}\right)=1$ atm, 1 equiv. ${ }^{\mathrm{t}} \mathrm{BuOH}\left[{ }^{\mathrm{t}} \mathrm{BuOH}\right]_{0}=0.282 \mathrm{mM}$, spectra recorded every $0.5 \mathrm{~s}$ for $15 \mathrm{~s}$, (c) 5 and $\mathrm{H}_{2}$ to 6. Conditions: Toluene solvent, [5 $]_{0}=0.167 \mathrm{mM}, \mathrm{p}\left(\mathrm{H}_{2}\right)=760 \mathrm{mmHg}$, 8 equiv. ${ }^{\mathrm{t}} \mathrm{BuOH}\left(\left[{ }^{\mathrm{t}} \mathrm{BuOH}\right]_{0}=1.33 \mathrm{mM}\right)$, spectra recorded every $0.5 \mathrm{~s}$ for $35 \mathrm{~s}$.

Once rate constants for the unshuttled reaction were obtained, reaction profiles in the presence of additives were modelled as two reactions running simultaneously, one according to Eq. 1 and a second according to Eq. 3. In this case, values of $k_{1}$ and $k_{-1}$ were fixed to the value determined from the unshuttled reactions. For the value of $k_{-2}$, the COPASI program was instructed to fix the value according to Eq. 4, thus linking it to the freely-refining value of $k_{2}$. Allowing free refinement of both $k_{1}$ and $k_{2}$ simultaneously gave rate constant values for the unshuttled reaction that were similar to those obtained from modelling only the unshuttled kinetic profiles but that were highly correlated to the shuttled rate constants. As a result, this modelling method was not used. Using the method described above, only one parameter was refined at a time, preventing correlation in 
the free refinement. In general, individual rate constants were assigned a $10 \%$ uncertainty (unless otherwise noted) in order to encompass errors due to measurement of reagents, systematic errors in spectroscopy, and uncertainty due to kinetic models.

\begin{tabular}{|c|c|}
\hline Reaction & $\lambda_{\max }(\varepsilon)$ \\
\hline $\mathbf{1}+\mathrm{H}_{2} \rightarrow \mathbf{2}$ & $522 \mathrm{~nm}\left(900 \mathrm{M}^{-1} \mathrm{~cm}^{-1}\right)$ \\
\hline $\mathbf{3}+\mathrm{H}_{2} \rightarrow \mathbf{4}$ & $309 \mathrm{~nm}\left(3000 \mathrm{M}^{-1} \mathrm{~cm}^{-1}\right)$ \\
\hline $\mathbf{5}+\mathrm{H}_{2} \rightarrow \mathbf{6}$ & $325 \mathrm{~nm}\left(10970 \mathrm{M}^{-1} \mathrm{~cm}^{-1}\right)$ \\
\hline
\end{tabular}

Table S2: Wavelengths and molar absorptivities used to determine concentration profiles for reactions monitored.

$$
\begin{gathered}
\mathrm{A}+\mathrm{B} \frac{k_{1}}{k_{-1}} \mathrm{C} \\
k_{-1}=k_{1} / K_{e q} \\
\mathrm{~A}+\mathrm{B}+\mathrm{D} \frac{k_{2}}{k_{-2}} \mathrm{C}+\mathrm{D} \\
k_{-2}=k_{2} / K_{e q}
\end{gathered}
$$

The variables of equations 1-3 are defined as follows: $\mathrm{A}=\mathbf{1}$ (or appropriate metal complex); $\mathrm{B}=$ $\mathrm{H}_{2} ; \mathrm{C}=\mathbf{2}$ (or appropriate metal complex); $\mathrm{D}=$ shuttle; $k_{1}=$ elementary rate constant for the forward reaction, addition of $\mathrm{H}_{2} ; k_{-1}$ = elementary rate constant for the reverse reaction, elimination of $\mathrm{H}_{2}$; $k_{2}=$ elementary rate constant for the addition of $\mathrm{H}_{2}$ mediated by a proton shuttle or additive; $k_{-2}=$ elementary rate constant for the elimination of $\mathrm{H}_{2}$ mediated by a proton shuttle or additive. 


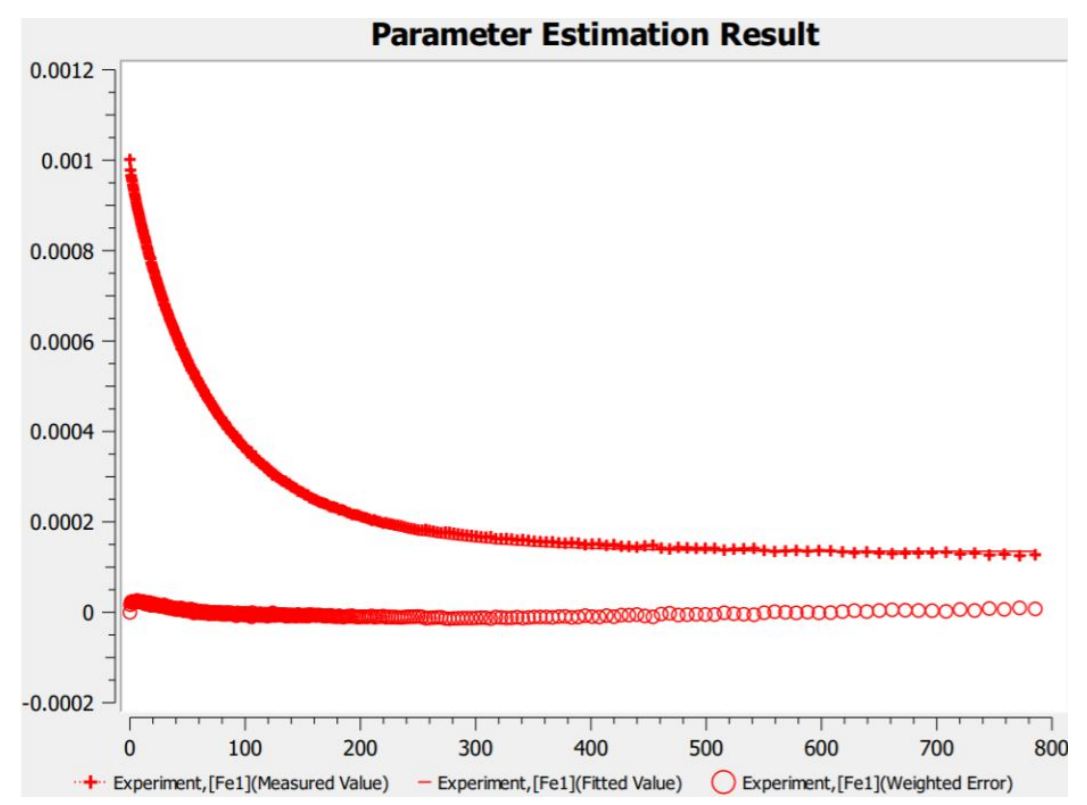

Figure S5: Representative concentration profile for the conversion of $\mathbf{1}$ and $\mathrm{H}_{2}$ to 2. Data points as + , residual error plotted as ${ }^{\circ}$. [1] in $\mathrm{mM}$ is plotted on the ordinate and time in seconds on the abscissa. Fit generated by COPASI drawn as a line. Reaction conditions: $[\mathbf{1}]_{0}=1.00 \mathrm{mM}$, under 1 atm of $\mathrm{H}_{2}$ at $20^{\circ} \mathrm{C}$ in toluene.

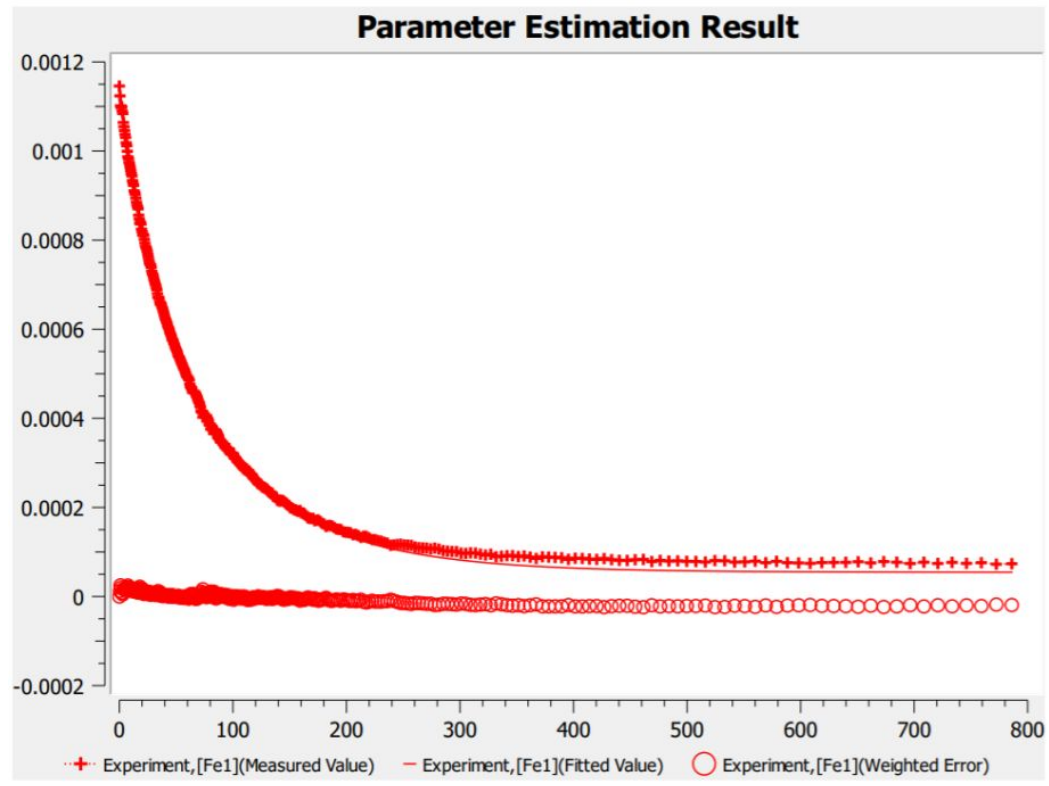

Figure S6: Representative concentration profile for the conversion of $\mathbf{1}$ and $\mathrm{H}_{2}$ to $\mathbf{2}$ in the presence of 8.0 equiv. of $\mathrm{BuOH}^{\mathrm{B}}$. Data points as + , residual error plotted as ${ }^{\circ}$. [1] in $\mathrm{mM}$ is plotted on the ordinate and time in seconds on the abscissa. Fit generated by COPASI drawn as a line. Reaction conditions: $[1]_{0}=1.80 \mathrm{mM}$, under 1 atm of $\mathrm{H}_{2}$ at $0{ }^{\circ} \mathrm{C}$ with $\left[{ }^{\mathrm{t}} \mathrm{BuOH}\right]_{0}=14.4 \mathrm{mM}$ in toluene. 
SIV. Empirical Rate Law Determination for Conversion of 1 to $\mathbf{2}$

In order to confirm that the reaction models used in numerical modelling were valid, the rate laws for the desired reactions were empirically determined. The rate law for the conversion of $\mathbf{1}$ to $\mathbf{2}$ in the presence of $\mathrm{H}_{2}$ was determined by altering the initial concentrations of each starting material independently and determining the dependence of the observed rate constant, $k_{o b s}$, for those reaction sets. The unshuttled and shuttled reactions conformed to the rate laws defined by Eq. 1 and Eq. 3, respectively (See Section SIII).

Rate dependence data for the reaction of $\mathbf{1}$ with $\mathrm{H}_{2}$ to form $\mathbf{2}$ in the absence of additives:

Rate Dependence on Initial Concentration of 1

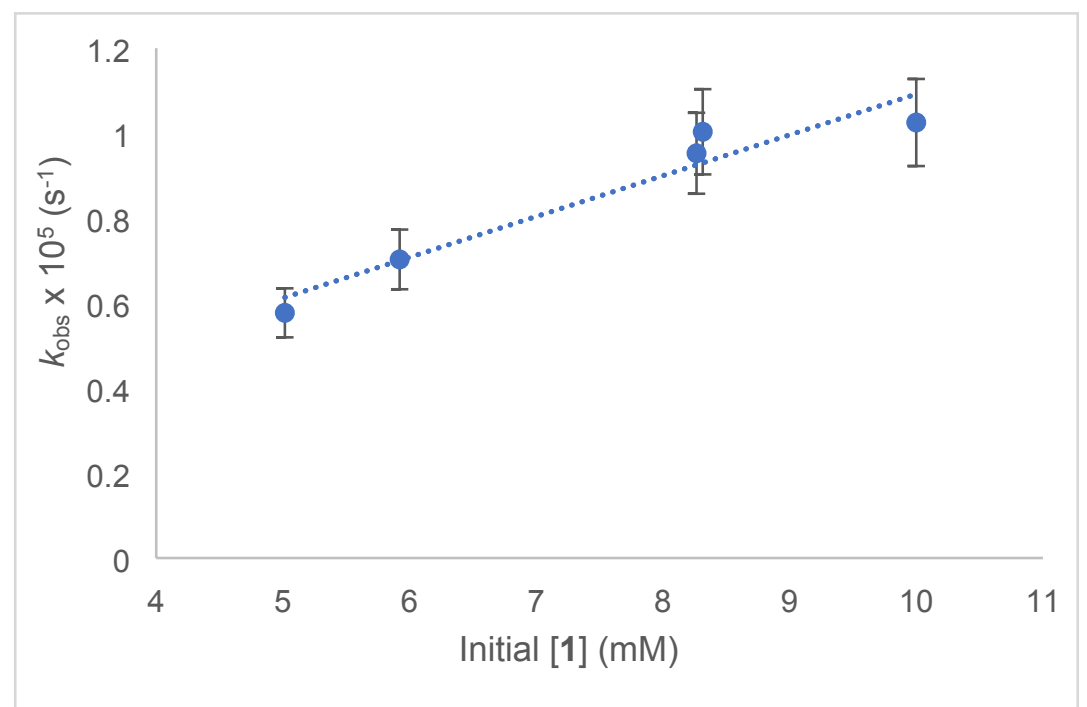

Figure S7: Dependence of $k_{o b s}$ on initial concentration of $\mathbf{1}$ as determined by the method of initial rates.

Rate Dependence on Partial Pressure of $\mathrm{H}_{2}$

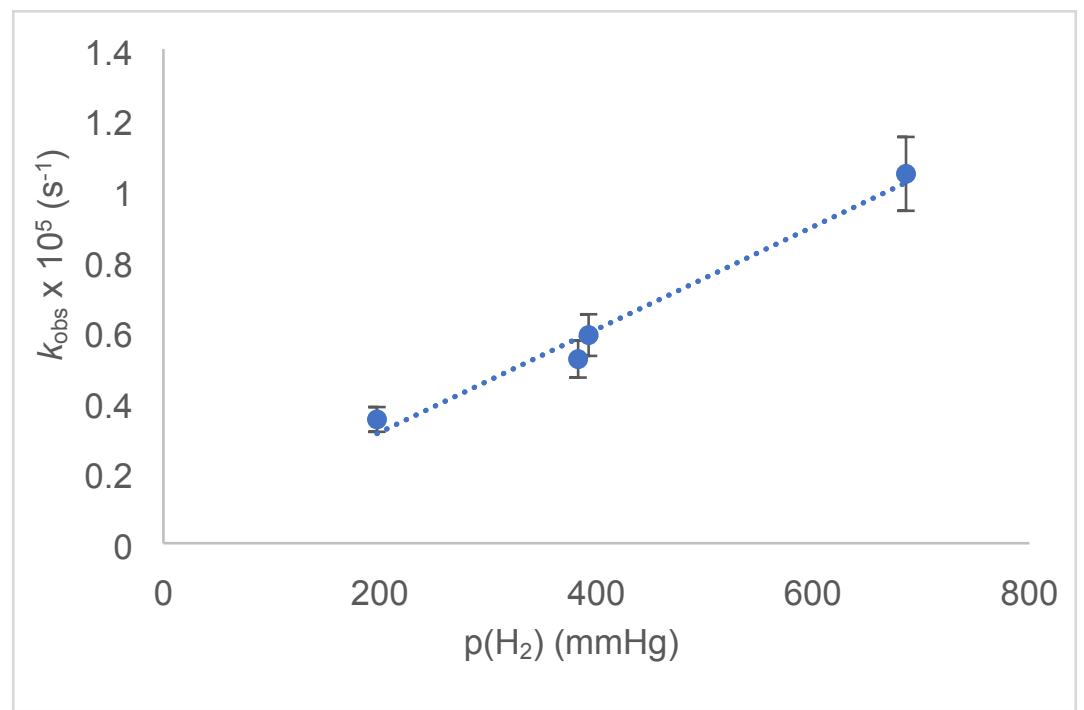

Figure S8: Dependence of $k_{o b s}$ on partial pressure of $\mathrm{H}_{2}$ as determined by the method of initial rates. 
$\underline{\text { Rate dependence data for reaction of } 1 \text { and } \mathrm{H}_{2}}$ to 2 in the presence of ${ }^{\mathrm{B}} \mathrm{BuOH}$ :

Rate Dependence on Initial Concentration of 1

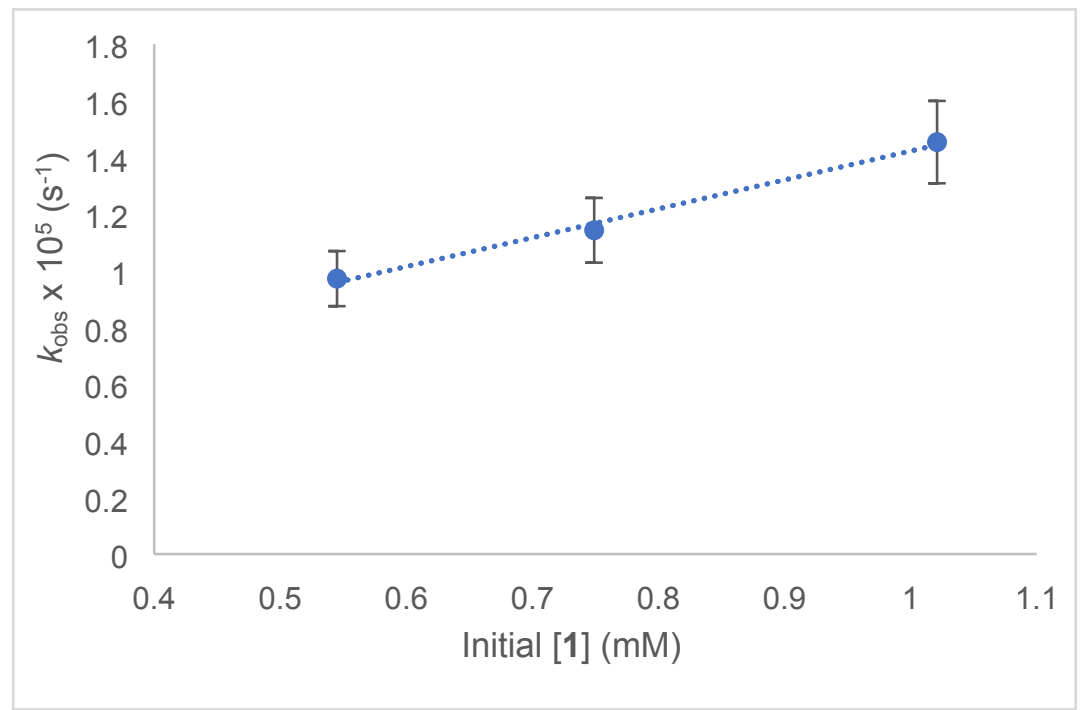

Figure S9: Dependence of $k_{o b s}$ on initial concentration of $\mathbf{1}$ in the presence of 10 equiv. of ${ }^{t} \mathrm{BuOH}$ and $1 \mathrm{~atm}$ of $\mathrm{H}_{2}$ as determined by the method of initial rates.

\section{Rate Dependence on Initial Concentration of ${ }^{\mathrm{t}} \mathrm{BuOH}$}

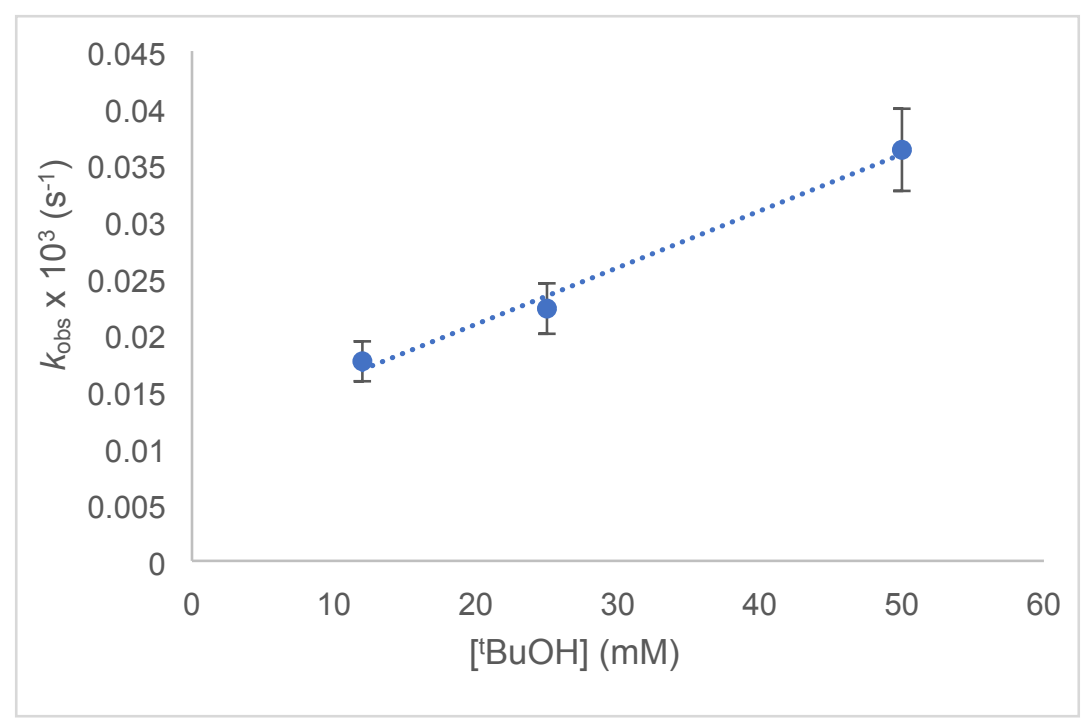

Figure S10: Dependence of $k_{\text {obs }}$ on concentration of ${ }^{\mathrm{t}} \mathrm{BuOH}$ with initial concentration of $\mathbf{1}$ as $1.0 \mathrm{mM}$ and $1 \mathrm{~atm}$ of $\mathrm{H}_{2}$ as determined by the method of initial rates. 


\section{Rate Dependence on Partial Pressure of $\mathrm{H}_{2}$}

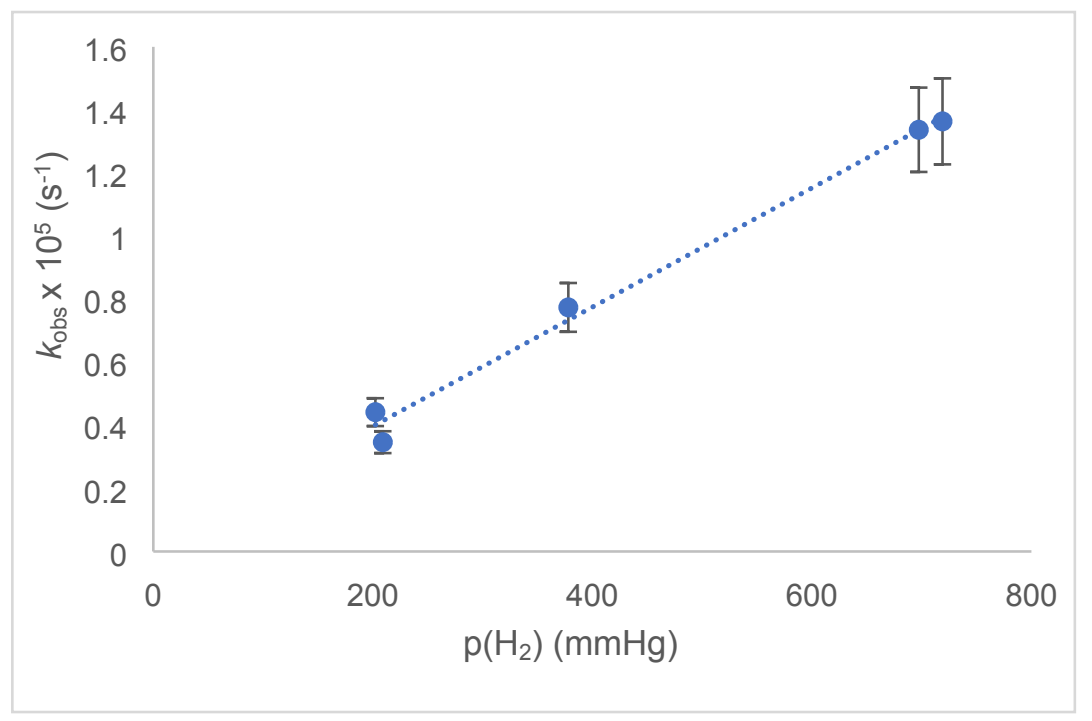

Figure S11: Dependence of $k_{o b s}$ on partial pressure of $\mathrm{H}_{2}$ with 10 equiv, ${ }^{\mathrm{t}} \mathrm{BuOH}$ and an initial concentration of 1 of $1.0 \mathrm{mM}$ as determined by the method of initial rates. 


\section{SV. Eyring Plots for the Conversion of 1 to 2}

Shown below are the Eyring plots used to obtain the activation parameters for both the unshuttled and shuttled rate constants ( $k_{l}$ and $k_{2}$, respectively). Rate constants were determined using COPASI modelling for each data point (vide supra). Reaction conditions were $1 \mathrm{~atm}$ of $\mathrm{H}_{2}, 1.00 \mathrm{mM}$ for $[1]_{0}$, at temperatures which ranged from $243 \mathrm{~K}$ to $298 \mathrm{~K}$. The error bars pictured are $10 \%$ error bars for each data point. The Eyring plot for $k_{2}$ has a poor fit as reflected by the relatively low value of $\mathrm{R}^{2}$. This is attributed in part to the fact that there is only a very small change in the rate constant for the forward shuttled reaction even when there are large changes in temperatures (likely, this is caused by the very small enthalpy of activation). As a result the slope of the line is very shallow and we are attempting to measure small differences, which introduces larger relative systematic error. However, several points do not lie on the line even when the standard error assigned to the value is considered. Therefore, the reported activation parameters for values of $k_{2}$ are reported with relatively large error bars. The values of $k_{-2}$ were obtained from the values of $k_{2}$ and the equilibrium constant, $\mathrm{K}_{\mathrm{eq}}$, determined from van't Hoff analysis (See Sections SIII and SVII). Even though the low quality of the data used to obtain $k_{2}$ will also affect $k_{-2}$, the contribution of $k_{2}$ is small, relative to the contribution from $\mathrm{K}_{\mathrm{eq}}$. As such, the linearity of the Eyring plot used to determine $k_{-2}$ is excellent. This is in agreement with our hypothesis that the relatively small change in the rate constant with temperature is giving rise to the problems in measuring $k_{2}$.

\section{Eyring $k_{1}$}

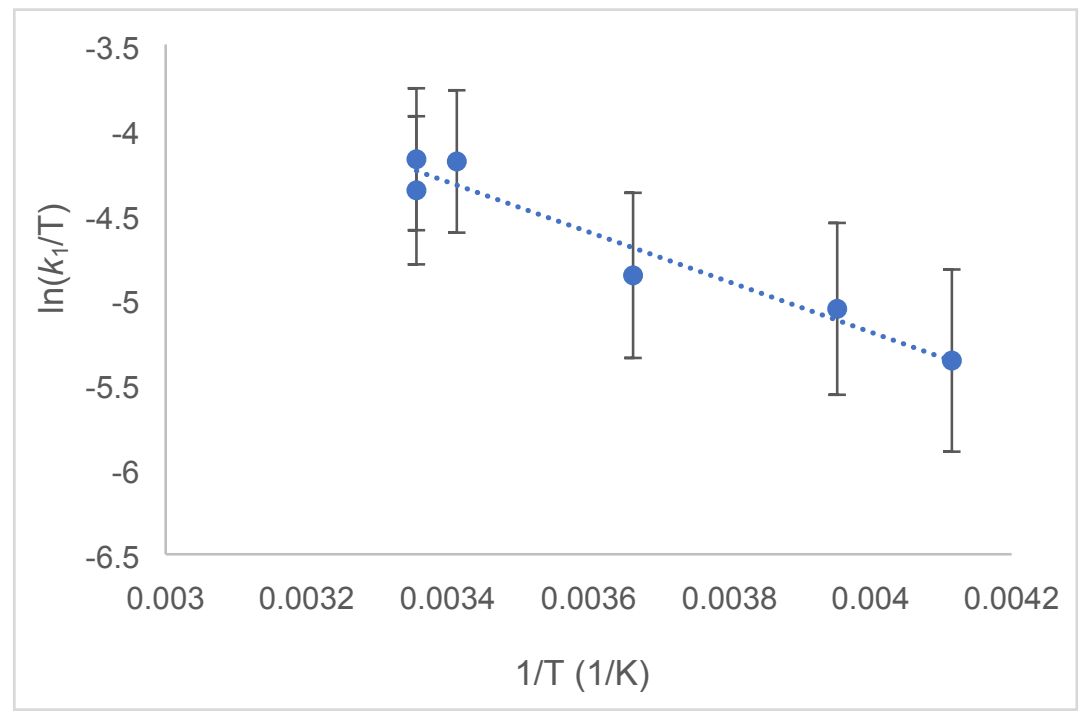

Figure S12: Eyring Plot for the rate constant $k_{1}$, unshuttled formation of $\mathbf{2}$ from $\mathbf{1}$ and $\mathrm{H}_{2}$. 


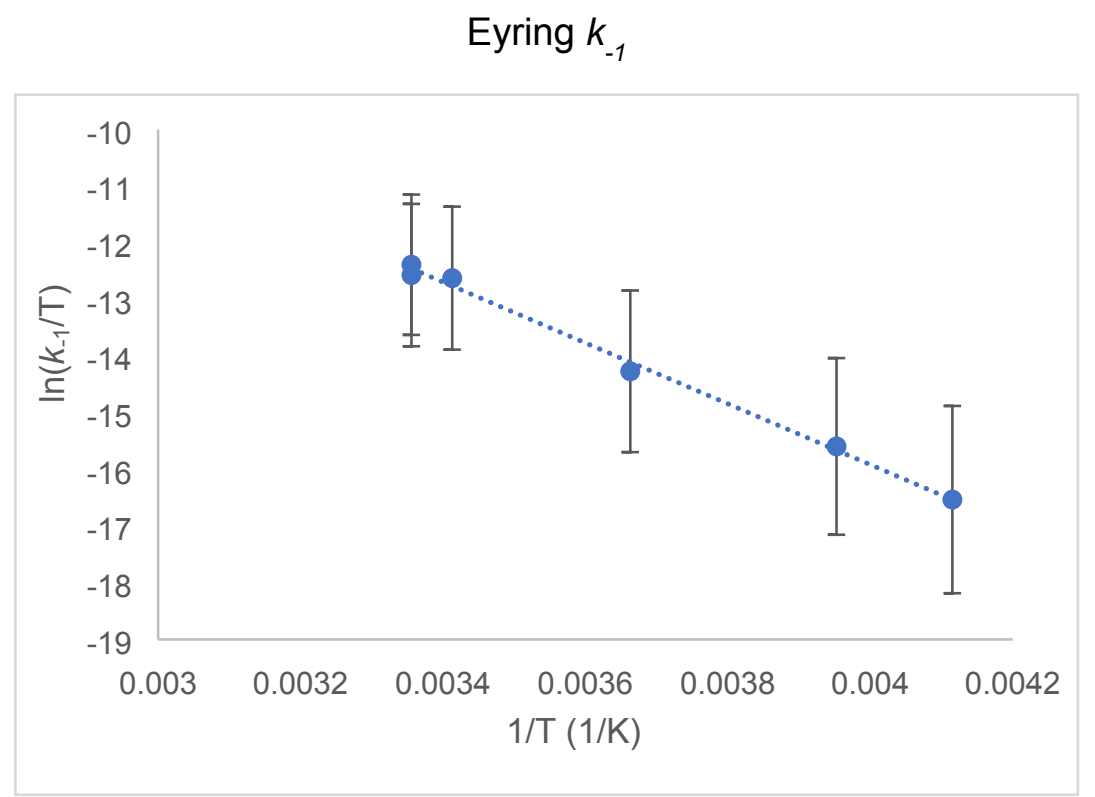

Figure S13: Eyring Plot for the rate constant $k_{-1}$, unshuttled formation of $\mathbf{1}$ and $\mathrm{H}_{2}$ from 2.

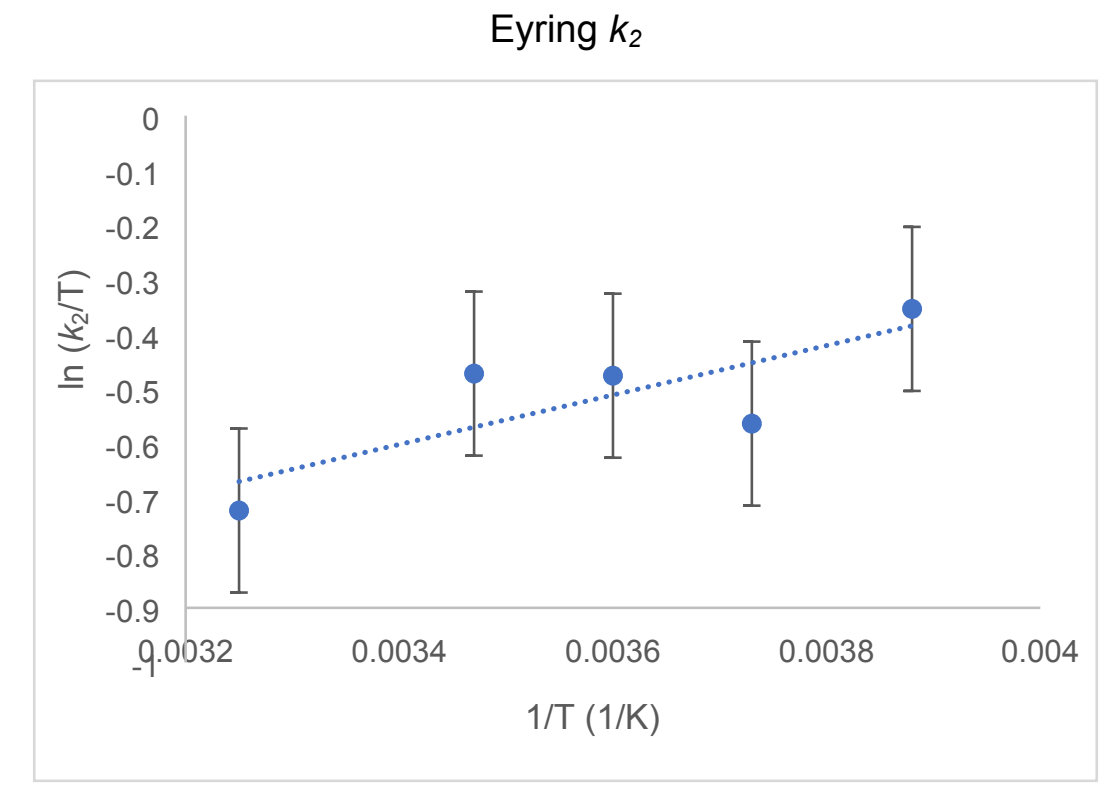

Figure S14: Eyring Plot for the rate constant $k_{2}$, formation of $\mathbf{2}$ from $\mathbf{1}$ and $\mathrm{H}_{2}$ in the presence of 8 equiv. of ${ }^{\text {t}} \mathrm{BuOH}$. 


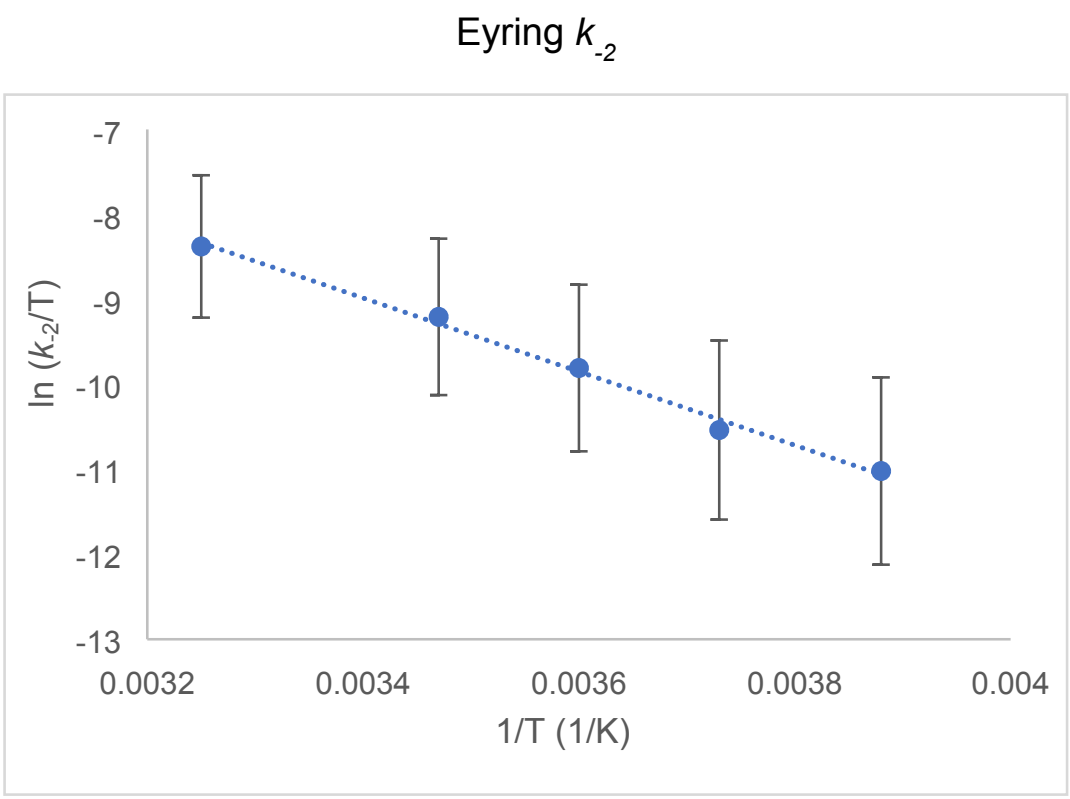

Figure S15: Eyring Plot for the rate constant $k_{-2}$, formation of $\mathbf{1}$ and $\mathrm{H}_{2}$ from $\mathbf{2}$ in the presence of 8 eqiuv. of ${ }^{\mathrm{BuOH}}$. 
SVI. Kinetic Isotope Effect Measurements for the Conversion of 1 to 2

Reagents that were commercially available $\left(\mathrm{D}_{2}\right.$ and $\left.{ }^{\mathrm{t}} \mathrm{BuOD}\right)$ were purchased as $\geq 99 \% \mathrm{D}$ incorporation and used as received. The deuteride complex 1-d $\boldsymbol{d}_{\mathbf{1}}$ was synthesized according to a modified procedure for the protio congener, $1 .{ }^{2}$ This included the synthesis of an intermediate, the deuterio-chloride complex (Scheme S2), which was also synthesized according to a modified literature procedure for the protio congener and used without isolation. ${ }^{1}$

A Schlenk flask was charged with ( $\left.{ }^{\mathrm{iPr} P N}{ }^{\mathrm{H}} \mathrm{P}\right) \mathrm{Fe}(\mathrm{Cl})_{2}(\mathrm{CO})(150 \mathrm{mg}, 0.33 \mathrm{mmols}), \mathrm{NaBD}_{4}(14 \mathrm{mg}$, $0.32 \mathrm{mmols})$, benzene $(5 \mathrm{~mL})$, ethanol- $\boldsymbol{d}_{\mathbf{1}}(5 \mathrm{~mL})$, and a stir bar. Upon addition of the ethanol, the solution developed an orange color with concomitant gas evolution. The reaction mixture was stirred for 3 hours, after which the volatiles were removed in vacuo. The resultant orange solid, the crude deuterio-chloride complex (Scheme S2), was extracted with $3 \times 5 \mathrm{~mL}$ of toluene in order to remove the salt by-product. The solution of crude deuterio-chloride complex was transferred via cannula to a Schlenk flask containing KO'Bu ( $40 \mathrm{mg}, 0.36 \mathrm{mmols})$, a magnetic stir bar, and 15 $\mathrm{mL}$ of THF. Upon mixing, the solution developed a dark red color. After stirring for 15 minutes, the volatiles were removed in vacuo and the resultant solid was extracted with $3 \times 5 \mathrm{~mL}$ of pentane and the solvent removed in vacuo. The crude solid was recrystallized from a concentrated solution in pentane at $-35^{\circ} \mathrm{C}$. Purity was determined by both ${ }^{31} \mathrm{P}$ and ${ }^{1} \mathrm{H}$ NMR spectroscopy (Figure S15). Residual protio substitution was minimal $(\sim 2 \%)$.

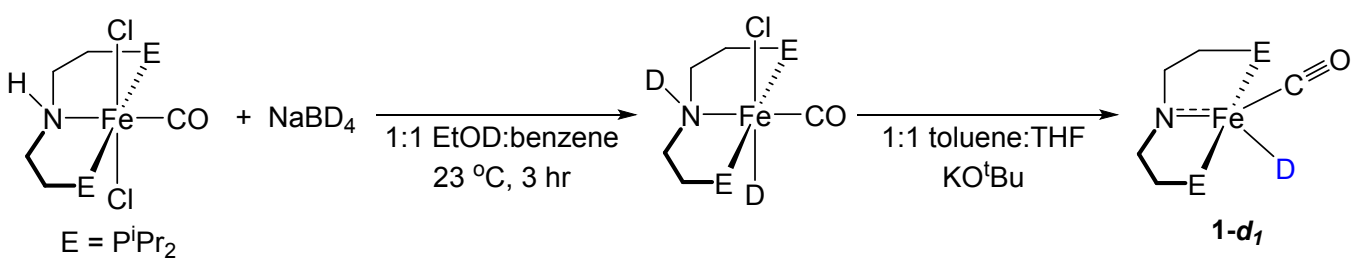

Scheme S2: Synthesis of $\mathbf{1}-\boldsymbol{d}_{\mathbf{1}}$.

${ }^{1} \mathrm{H}$ NMR spectrum of $\mathbf{1}-\boldsymbol{d}_{\mathbf{1}}$

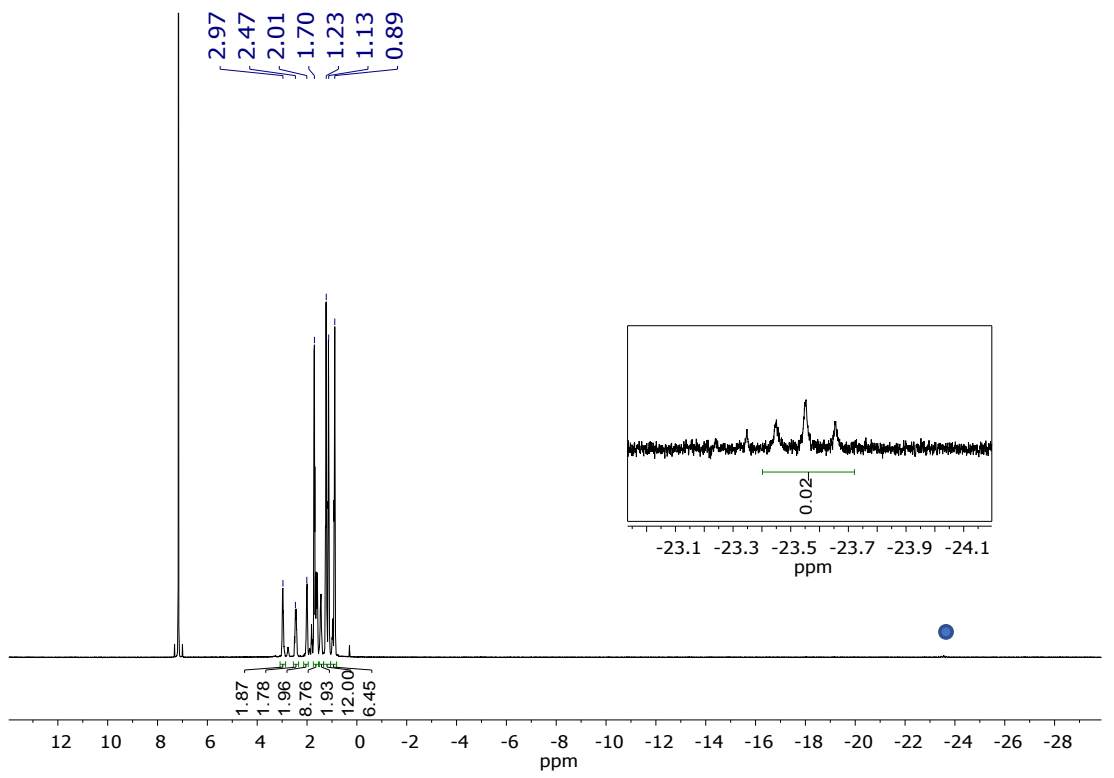

Figure S16: ${ }^{1} \mathrm{H}$ NMR spectrum to determine purity of $\mathbf{1 - d _ { 1 }}$. The location of residual hydride impurity is denoted by $\bullet$ and shown in the inset. 
Hydride/deuteride ligands on $\mathbf{2}$ are in chemical exchange with the N-H/D moiety, which is itself in chemical exchange with protic species such as ${ }^{\mathrm{t}} \mathrm{BuOH} / \mathrm{D}$. Due to this chemical exchange, isotopic substitution does not remain constant during the process of establishing equilibrium between $\mathbf{1}$ and $\mathrm{H}_{2}$ and $\mathbf{2}$. In the initial regime, however, where only a small amount of $\mathbf{2}$ is present, observable isotopic exchange is minimal. Additionally, the equilibrium between these species demonstrates strong equilibrium isotope effects (EIEs) wherein the value of $\mathrm{K}_{\mathrm{eq}}$ is dependent upon isotopic substitution (Scheme S3). Therefore, kinetic isotope effects (KIEs) were determined for the forward reaction $\left(\mathbf{1} / \mathbf{1}-\mathbf{d}_{\mathbf{1}}+\mathrm{H}_{2} / \mathrm{D}_{2} \rightarrow \mathbf{2}-\mathbf{d}_{\mathbf{n}}\right)$ via the method of initial rates. Under the given set of conditions, elementary rate constants were determined by dividing the $k_{\mathrm{obs}}$ determined from the method of initial rates by the initial concentrations of all relevant reagents. Because the rate is measured by two terms, the unshuttled and the shuttled term, if necessary, values of $k_{1, D}$ were determined for a given set of conditions first and then used to determine the appropriate values of $k_{2, D}$. which were then used in combination with the values of $k_{1}$ or $k_{2}$ determined from COPASI modelling of full kinetic traces to calculate the KIE values. Reaction conditions included $[1]_{0}=1.00$ $\mathrm{mM}, 1 \mathrm{~atm}$ of $\mathrm{H}_{2}$ or $\mathrm{D}_{2}$ combined as noted in the "UV-Vis and Analysis Methods" at $25^{\circ} \mathrm{C}$.

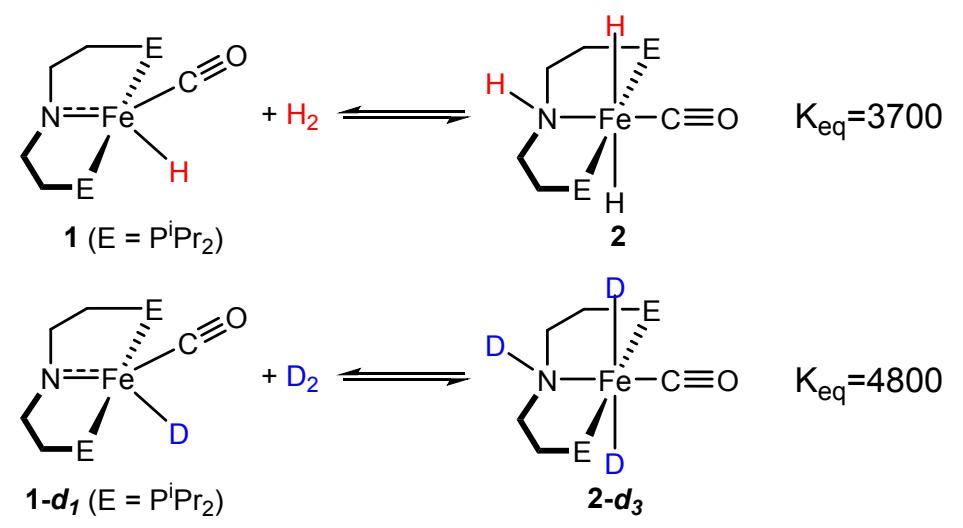

Scheme S3: An example of an EIE on the equilibrium between $\mathbf{1}-\boldsymbol{d}_{\mathbf{n}}, \mathrm{H}_{2}-\boldsymbol{d}_{m}$, and $\mathbf{2}-\boldsymbol{d}_{\mathbf{m}+\mathbf{n}}$. Protio equilibrium constant determined from van't Hoff plot (see below). Deuterio equilibrium contant also determined from van't Hoff plot, $1 \mathrm{~atm}$ of $\mathrm{D}_{2}$, total $[\mathrm{Fe}]=11.5 \mathrm{mM}$.

Discussion of the Impact of Bond Enthalpies on the Kinetic Isotope Effect(s) of the Shuttled Reaction: This discussion makes use of entry titles from Table 4 in the main text of the paper. The bonds broken and formed in each transition state are given below in the tables for Entries 3 (Table S3), 4 (Table S4), and 5 (Table S5) of Table 4 of the main text. If the kinetic isotope effect is dominated by enthalpic terms, the KIE should be proportional to the differences in bond zero-point energies that can be derived from their stretching frequencies. This traditional interpretation of KIEs is difficult to apply in this scenario due to the fact that the strength of the $\mathrm{Fe}-\mathrm{N} \pi$-bond being broken is uncertain. However, relative kinetic isotope effects can at least be described on a qualitative basis by considering relative bond enthalpies.

In Entry 3, an O-D bond is broken in the transition state and a new N-D bond is formed. However, a new $\mathrm{O}-\mathrm{D}$ bond is also formed. As a result, the net bonds formed and broken in Entry 3 are: an $\mathrm{Fe}-\mathrm{N} \pi$-bond and $\mathrm{D}-\mathrm{D}$ bond are broken, and an $\mathrm{N}-\mathrm{D}$ and $\mathrm{Fe}-\mathrm{D}$ bond are formed. For Entry 4, however, the $\mathrm{O}-\mathrm{D}$ bond is replaced by an $\mathrm{O}-\mathrm{H}$ bond, as it is an $\mathrm{H}-\mathrm{H}$ bond that is breaking instead of a D-D bond. Because deuterium has an energetic preference to be in the strongest bond, the transfer of a deuterium to nitrogen in Entry 4 causes a change in reaction enthalpy in the positive direction relative to Entry 3. In contrast, in Entry 5 there is no obvious preference for deuterium to 
occupy a certain position in either the starting material or products and as a result it gives a similar KIE to Entry 3.

Table S3: Bonds Broken and Formed in the Transition State for Entry 3. ${ }^{a}$

\begin{tabular}{cc}
\hline Bonds Broken & Bonds Formed \\
\hline Fe-N $(\pi)$ & N-D \\
O-D & O-D \\
D-D & Fe-D \\
\hline
\end{tabular}

${ }^{a} k_{\mathrm{H}} / k_{\mathrm{D}}=1.7 \pm 0.2$ for $\mathrm{D}_{2}$ and ${ }^{\mathrm{t}} \mathrm{BuOD}\left(\mathrm{vs} . \mathrm{H}_{2}\right.$ and $\left.{ }^{\mathrm{t}} \mathrm{BuOH}\right)$.

Table S4: Bonds Broken and Formed in the Transition State for Entry $4 .^{a}$

\begin{tabular}{cc}
\hline Bonds Broken & Bonds Formed \\
\hline $\mathrm{Fe}-\mathrm{N}(\pi)$ & $\mathrm{N}-\mathrm{D}$ \\
$\mathrm{O}-\mathrm{D}$ & $\mathrm{O}-\mathrm{H}$ \\
$\mathrm{H}-\mathrm{H}$ & $\mathrm{Fe}-\mathrm{H}$ \\
\hline
\end{tabular}

${ }^{a} k_{\mathrm{H}} / k_{\mathrm{D}}=5.0 \pm 0.6$ for $\mathrm{H}_{2}$ and ${ }^{\mathrm{t}} \mathrm{BuOD}\left(\right.$ vs. $\mathrm{H}_{2}$ and $\left.{ }^{\mathrm{t}} \mathrm{BuOH}\right)$.

Table S5: Bonds Broken and Formed in the Transition State for Entry 5. ${ }^{a}$

\begin{tabular}{cc}
\hline Bonds Broken & Bonds Formed \\
\hline $\mathrm{Fe}-\mathrm{N}(\pi)$ & $\mathrm{N}-\mathrm{H}$ \\
$\mathrm{O}-\mathrm{H}$ & $\mathrm{O}-\mathrm{D}$ \\
$\mathrm{D}-\mathrm{D}$ & $\mathrm{Fe}-\mathrm{D}$ \\
${ }^{a} k_{\mathrm{H}} / k_{\mathrm{D}}=1.8 \pm 0.2$ for $\mathrm{D}_{2}$ and ${ }^{\mathrm{t}} \mathrm{BuOH}\left(\right.$ vs. $\mathrm{H}_{2}$ and $\left.{ }^{\mathrm{t}} \mathrm{BuOH}\right)$. &
\end{tabular}


SVII. Van't Hoff Plots for Addition of $\mathrm{H}_{2}$ to 1

Measurements of the equilibrium constant $\mathrm{K}_{\mathrm{eq}}$ at different temperatures were made in order to generate van't Hoff plots for thermodynamic parameters of the reaction. This provided information on the favorability of the formation of $\mathbf{2}$ with a variety of different additives as well as reliable measurements of the equilibrium constant under different conditions for kinetic modelling. The plots made using ${ }^{1} \mathrm{H}$ NMR spectroscopy are given below. Equilibrium constants were within error of the observed equilibrium constant in kinetic trials as determined by the concentration of $\mathbf{1}$ remaining at the end of the reaction and assuming mass balance with no diffusion of $\mathrm{H}_{2}$ from the headspace. Measurements of $\mathrm{K}_{\text {eq }}$ by ${ }^{1} \mathrm{H}$ NMR spectroscopy were based on integrations of the hydride peaks for the relevant complexes relative to an internal standard (naphthalene). The concentration of the dihydride was recorded as the sum of the concentrations of the two isomers of $\mathbf{2}$ reported previously, as there should be no difference in the ability of the isomers to hydrogen bond to additives. ${ }^{2}$ If this was an issue, it would manifest as nonlinearity in the observed van't Hoff data.

Sample Procedure for Determination of a van't Hoff Plot: In a glovebox, a solution of 1 (5.9 $\mathrm{mg}, 0.015 \mathrm{mmols})$ was made in toluene- $d_{8}(600 \mu \mathrm{L})$. To this was added naphthalene $(10.2 \mathrm{mg})$ and, if applicable, the desired additive (for example $p$-bromo-2-phenyl-2-propanol, $32.6 \mathrm{mg}, 0.152$ mmols, 10.0 equiv.) was added to the solution. The solution was subsequently transferred into a JYoung NMR tube, sealed, and removed from the glovebox. Using a Schlenk manifold filled with $\mathrm{H}_{2}$, the solution was subjected to three freeze-pump-thaw cycles and the headspace of the NMR tube filled with $\mathrm{H}_{2}$. The tube was resealed and removed from the Schlenk manifold. The solution was shaken vigorously and allowed to set for 10 minutes. After insertion into the NMR probe, the temperature was regulated and the sample allowed to equilibrate for 10 minutes in between spectra. ${ }^{1} \mathrm{H}$ NMR spectra were taken with $16 \mathrm{scans}$, a delay time of $10 \mathrm{~s}$, and an acquisition time of $3.000 \mathrm{~s}$. ${ }^{31} \mathrm{P}$ NMR spectra were taken in order to monitor the sample for decomposition.

\section{Van't Hoff without Additives}

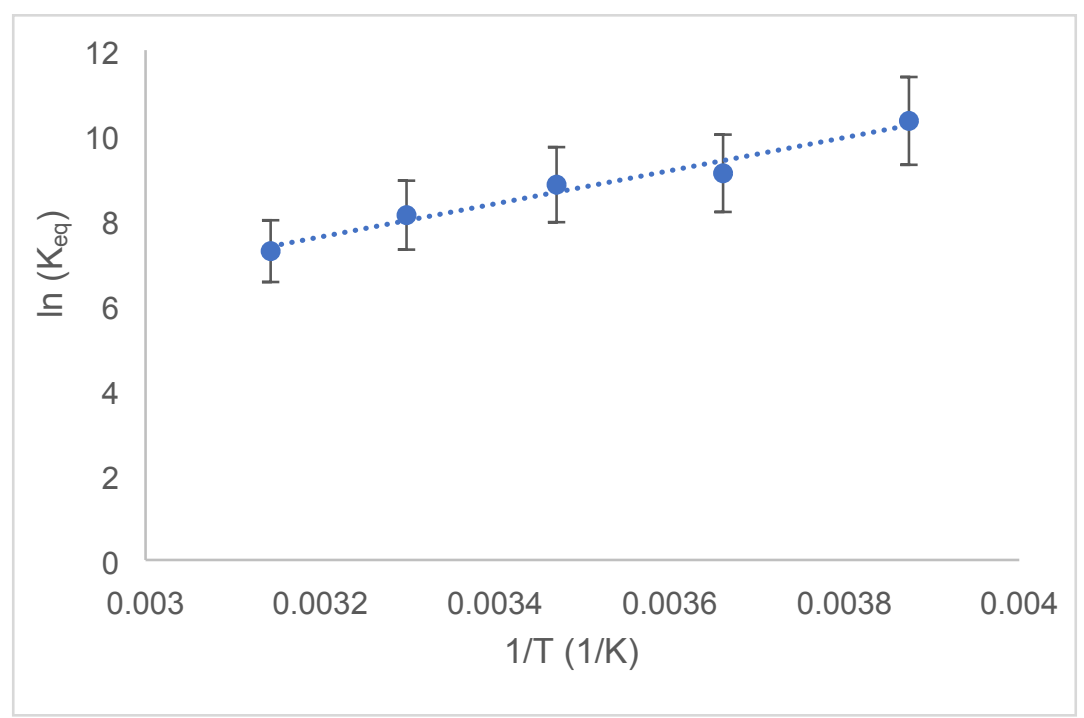

Figure S17: Van't Hoff plot measured by ${ }^{1} \mathrm{H}$ NMR spectroscopy for the equilibrium of $\mathbf{1}, \mathrm{H}_{2}$, and 2 with total $[\mathrm{Fe}]=20 \mathrm{mM}$. 


\section{Van't Hoff with 'BuOH}

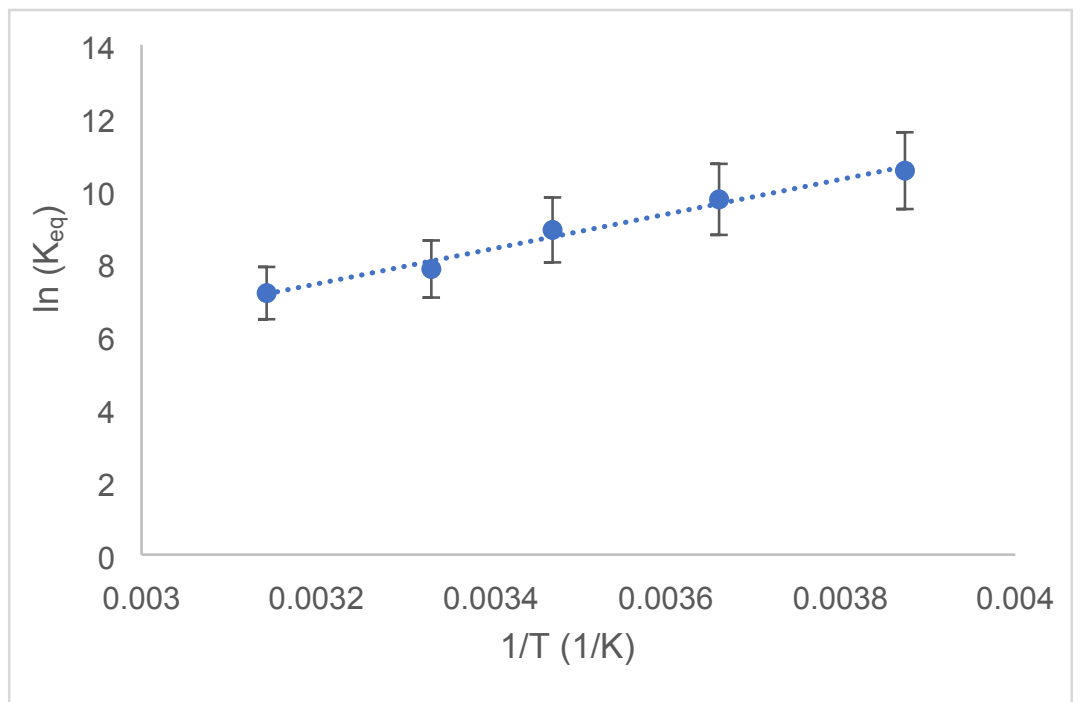

Figure S18: Van't Hoff plot measured by ${ }^{1} \mathrm{H}$ NMR spectroscopy for the equilibrium of $\mathbf{1}, \mathrm{H}_{2}$, and 2 , total $[\mathrm{Fe}]=15.2 \mathrm{mM}$, in the presence of 10 equiv. of ${ }^{\mathrm{t}} \mathrm{BuOH}(150 \mathrm{mM})$.

Van't Hoff with 2-phenyl-2-propanol

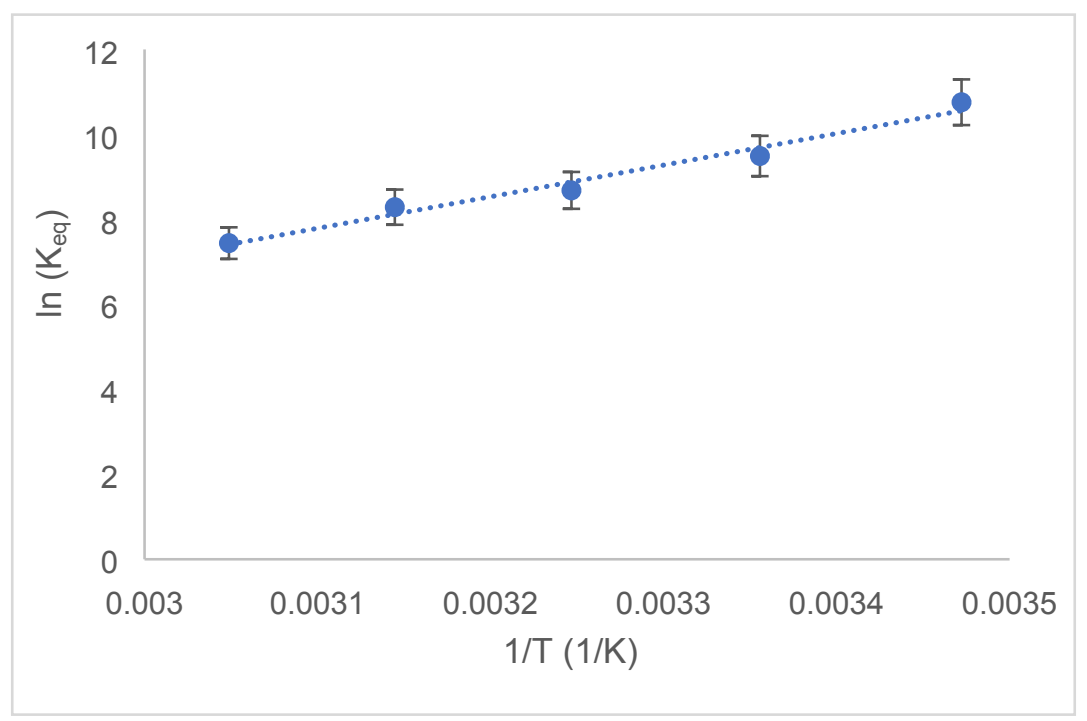

Figure S19: Van't Hoff plot measured by ${ }^{1} \mathrm{H}$ NMR spectroscopy for the equilibrium of $1, \mathrm{H}_{2}$, and 2 with total $[\mathrm{Fe}]=16 \mathrm{mM}$ in the presence of 10 equiv. of 2-phenyl-2-propanol (160 mM). 
Van't Hoff with p-bromo-2-phenyl-2-propanol

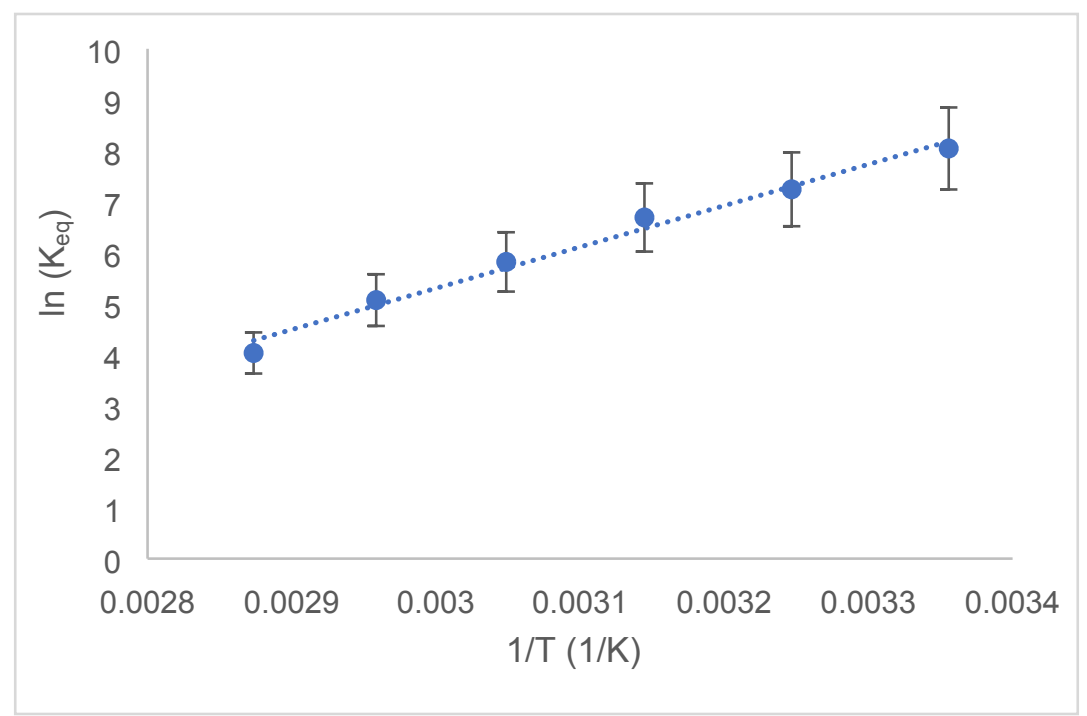

Figure S20: Van't Hoff plot measured by ${ }^{1} \mathrm{H}$ NMR spectroscopy for the equilibrium of $\mathbf{1}, \mathrm{H}_{2}$, and 2 with total $[\mathrm{Fe}]=25.3 \mathrm{mM}$ in the presence of 10 equiv. of $p$-bromo-2-phenyl-2-propanol (253 mM).

\section{Van't Hoff with p-methyl-2-phenyl-2-propanol}

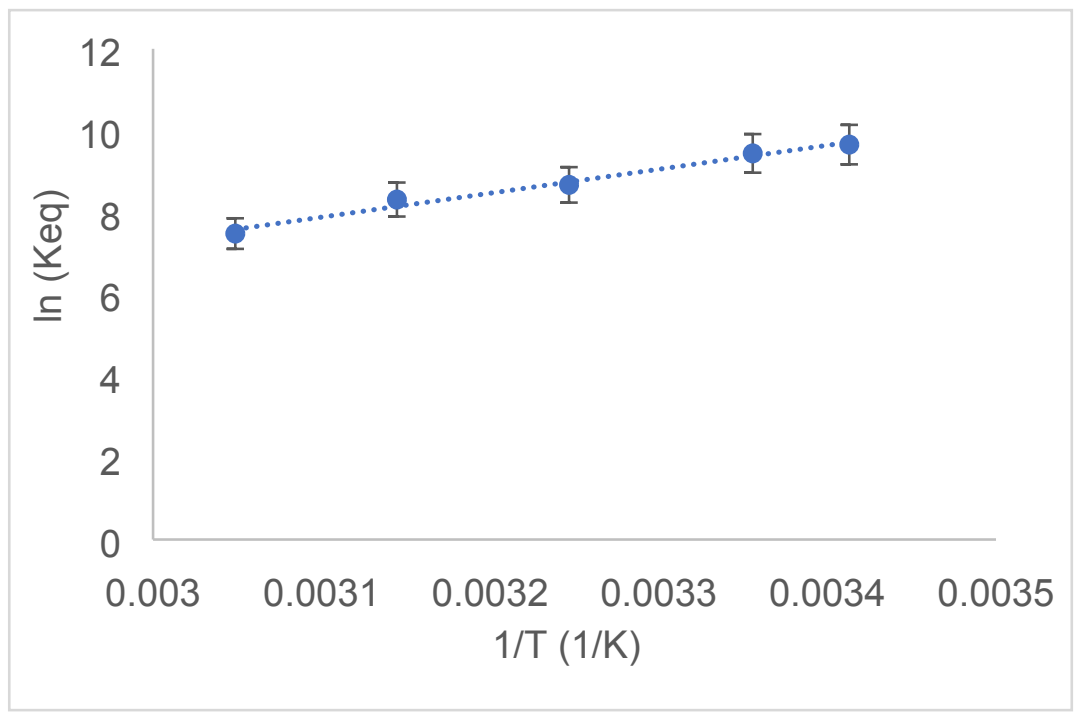

Figure S21: Van't Hoff plot measured by ${ }^{1} \mathrm{H}$ NMR spectroscopy for the equilibrium of 1, $\mathrm{H}_{2}$, and 2 with total $[\mathrm{Fe}]=18.2 \mathrm{mM}$ in the presence of 10 equiv. of $p$-methyl-2-phenyl-2-propanol $(180 \mathrm{mM})$. 


\section{Van't Hoff with MTBE}

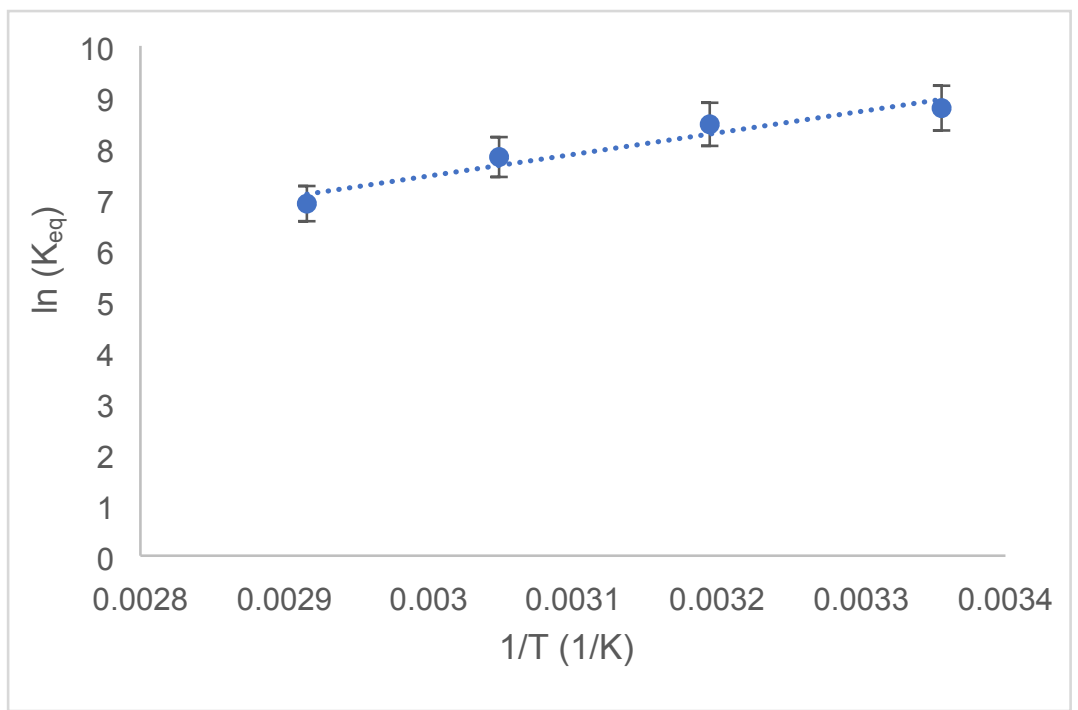

Figure S22: Van't Hoff plot measured by ${ }^{1} \mathrm{H}$ NMR spectroscopy for the equilibrium of $\mathbf{1}, \mathrm{H}_{2}$, and 2 with total $[\mathrm{Fe}]=47.9 \mathrm{mM}$ in the presence of 10 equiv. of methyl-tert-butyl ether (480 $\mathrm{mM})$.

Van't Hoff with 2,6-lutidine

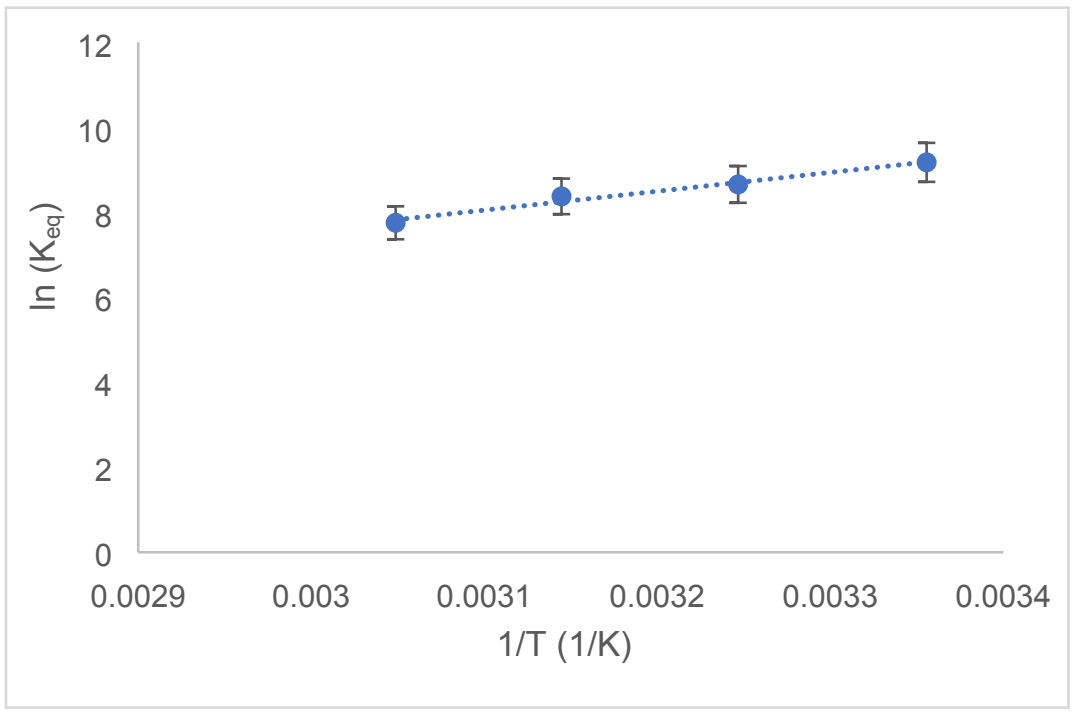

Figure S23: Van't Hoff plot measured by ${ }^{1} \mathrm{H}$ NMR spectroscopy for the equilibrium of $\mathbf{1}, \mathrm{H}_{2}$, and 2 with total $[\mathrm{Fe}]=30 \mathrm{mM}$ in the presence of 10 equiv. of 2,6-lutidine $(300 \mathrm{mM})$. 


\section{SVIII. Information on Hydrogen Bonding Between 2 and Reaction Additives}

For measurement of the strength of hydrogen bonding in the complex [2-Y] where $\mathrm{Y}$ is either a tertiary alcohol, 2,6-lutidine, or methyl-tert-butyl ether, the enthalpy of the reaction to form $\mathbf{2}$ from 1 and $\mathrm{H}_{2}$ was used instead of the Gibbs free energy. This is due to the fact that the equilibrium between these species in the presence of a hydrogen bonding partner is subject to a compensation effect, so the free energy does not provide the strength of the hydrogen bonding interaction (Figure S23). ${ }^{12}$ For demonstration purposes a plot of $\Delta \mathrm{G}_{298 \mathrm{~K}}$ from the van't Hoff plots vs. $k_{2}$, the shuttled rate constant, is shown below (Figure S24) and shows poor correlation.

\section{Compensation Effect of Enthalpy and Entropy}

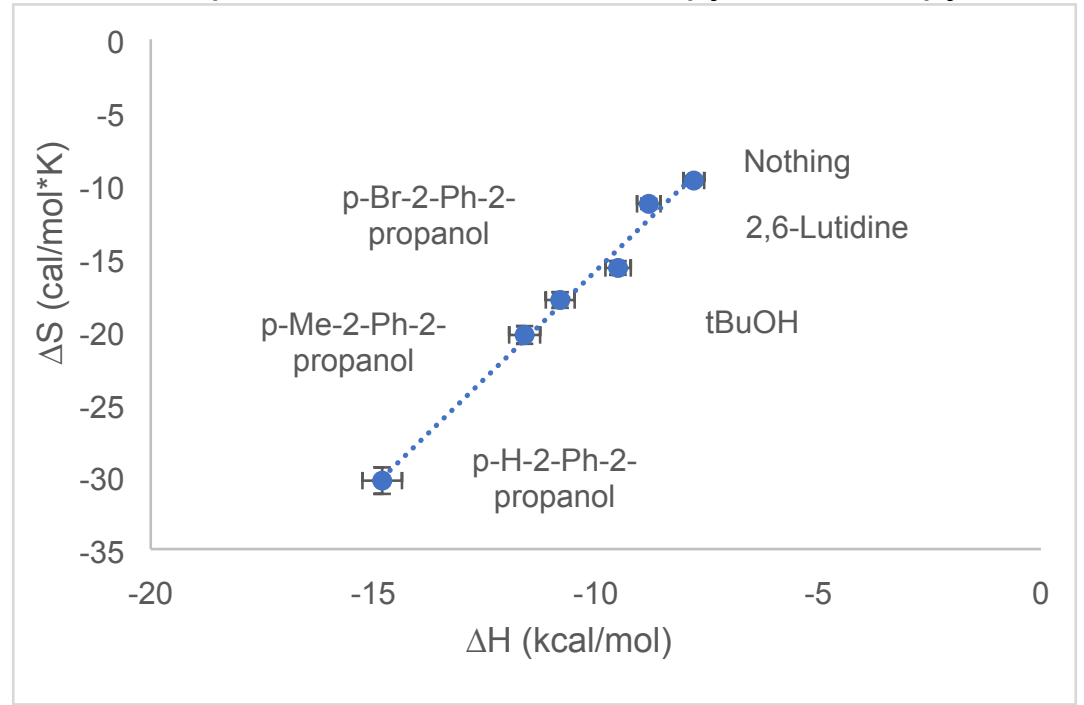

Figure S24: Compensation effect plot for the equilibrium of $\mathbf{1}$ and $\mathrm{H}_{2}$ and $\mathbf{2}$ based on values obtained from van't Hoff analysis in the preceding section.

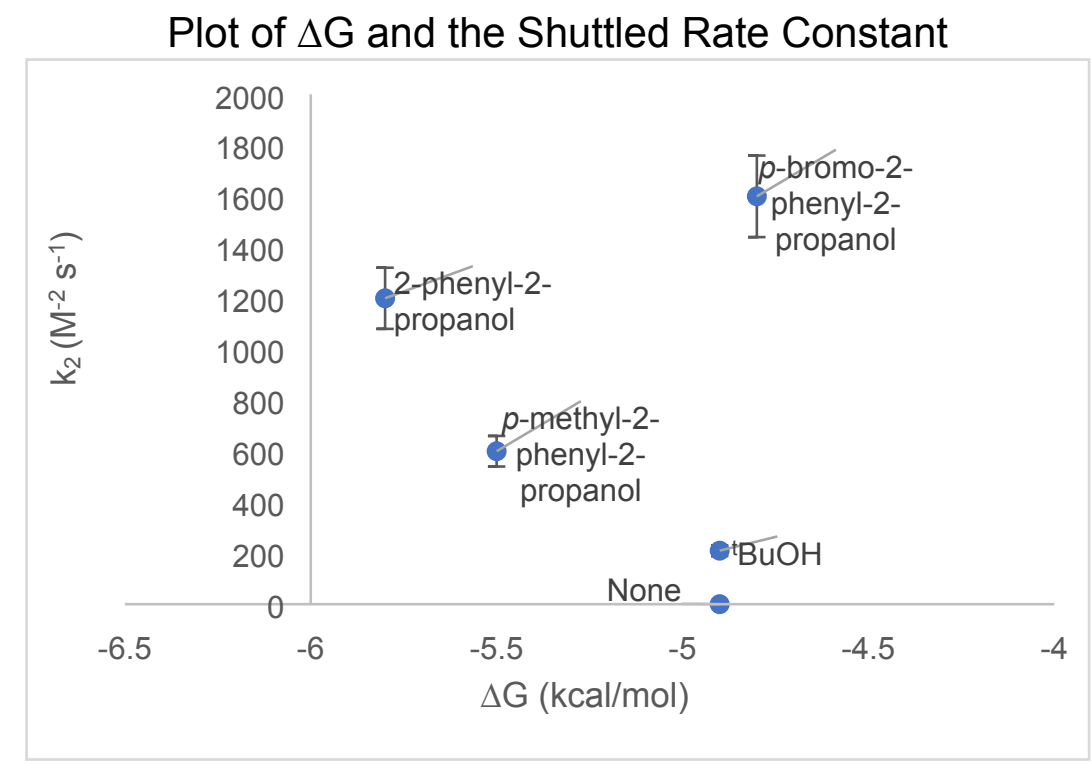

Figure S25: Scatter plot of the shuttled rate constant, $k_{2}$, vs. the free energy change of the reaction. There is no correlation. 
SIX. Infrared Spectroscopic Measurements of Complex 2 in the Presence of ${ }^{t} \mathrm{BuOH}$

Solution state infrared (IR) spectroscopy was used to observe the hydrogen bonding of ${ }^{\mathrm{B}} \mathrm{BuOH}$ to 2. The procedure for this experiment is given below. An overlay of the solution state IR spectra of a solution containing 2, 2 and ${ }^{\mathrm{t}} \mathrm{BuOH}$, and only ${ }^{\mathrm{t}} \mathrm{BuOH}$ are shown in Figure S25. A difference spectrum was generated by subtracting the spectrum of $\mathbf{2}$ and only ${ }^{\mathrm{t}} \mathrm{BuOH}$ from the spectrum of $\mathbf{2}$ and ${ }^{\mathrm{t}} \mathrm{BuOH}$ is shown in Figure $\mathrm{S} 26$.

For the spectra of 2, solutions were prepared as follows: A stock solution of $\mathbf{1}(8.9 \mathrm{mg}, 0.023$ mmols) in $\mathrm{C}_{6} \mathrm{D}_{6}(1.2 \mathrm{~mL})$ was prepared in a glovebox. An aliquot $(600 \mu \mathrm{L})$ of this solution was transferred to a Schlenk flask. To the remainder of the stock solution $(600 \mu \mathrm{L})$ was added ${ }^{\mathrm{t}} \mathrm{BuOH}$ ( $5 \mu \mathrm{L}, 0.05 \mathrm{mmols})$, and the solution transferred to a Schlenk flask. Both flasks were removed from the glovebox and attached to a Schlenk manifold. The solutions were subjected to three freeze-pump-thaw cycles, after which the flasks were refilled with $1 \mathrm{~atm}$ of $\mathrm{H}_{2}$ from the Schlenk manifold. After allowing the samples to equilibrate at room temperature for 10 minutes, each solution was transferred into a Hamilton SampleLock syringe, and after sealing the locking valve these syringes were moved into the glovebox containing the IR spectrometer.

For the spectrum of ${ }^{\mathrm{t}} \mathrm{BuOH}$, a stock solution of $5 \mu \mathrm{L}$ of ${ }^{\mathrm{t}} \mathrm{BuOH}(0.05 \mathrm{mmols})$ in $600 \mu \mathrm{L}$ of $\mathrm{C}_{6} \mathrm{D}_{6}$ was prepared in a glovebox, sealed in a Hamilton SampleLock syringe, and transferred directly to the glovebox containing the IR spectrometer.

For the measurement of a spectrum, a solution observation cell with a Luer lock inlet and sample chamber volume of approximately $300 \mu \mathrm{L}$ was used. The following was done for each of the two prepared solutions, the equilibrium mixture with and without ${ }^{t} \mathrm{BuOH}$, respectively: To measure the spectrum of a given solution, the SampleLock syringe was attached to the Luer lock inlet of the observation cell. Half of the solution in the syringe, $300 \mu \mathrm{L}$, was flushed through the observation chamber in order to purge it of $\mathrm{N}_{2}$ and any residual wash solvent. The remainder of volume in the syringe, $300 \mu \mathrm{L}$, was then injected into the observation chamber through the Luer lock inlet. The solution cell was then quickly sealed and a spectrum recorded. 


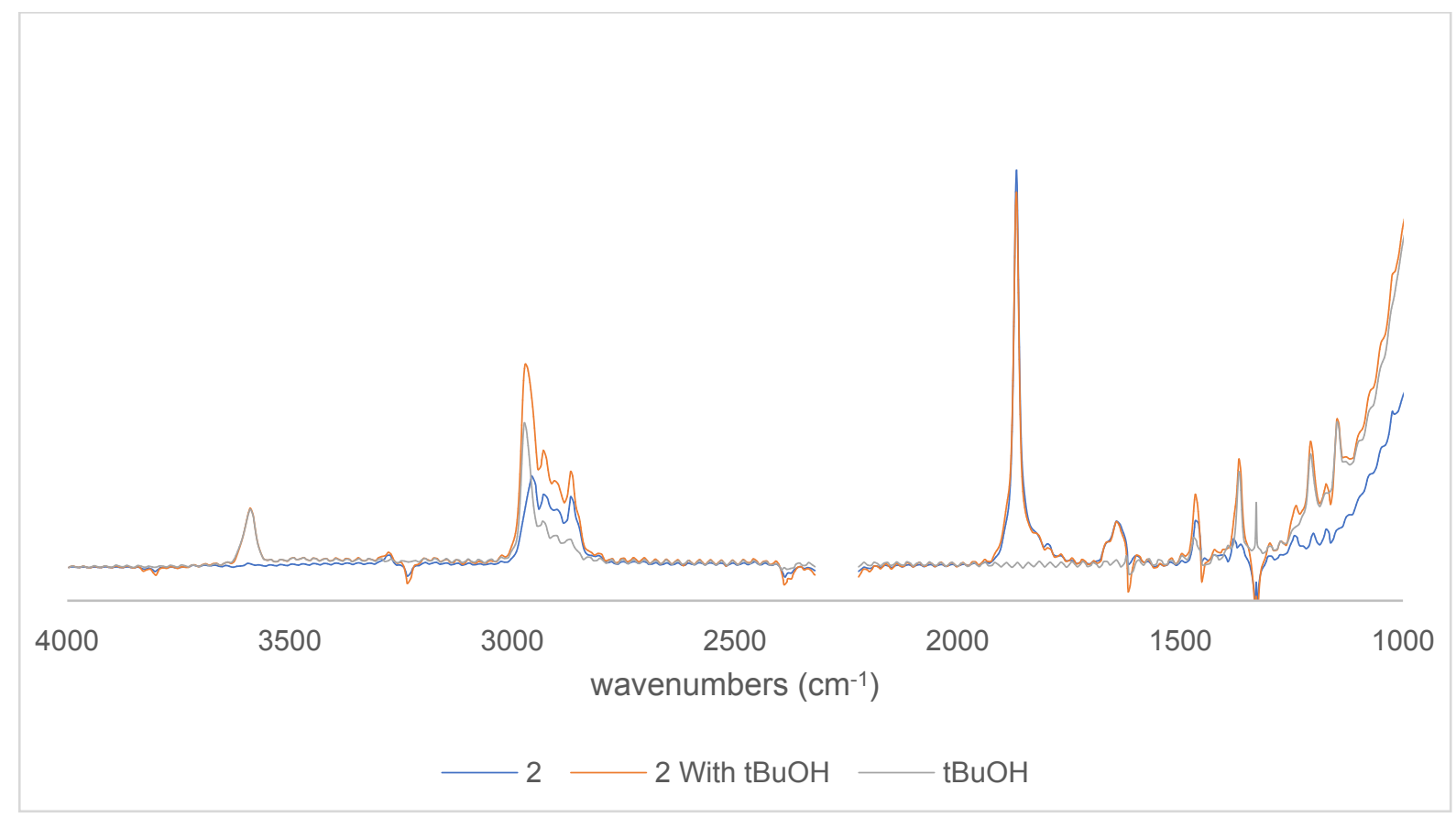

Figure S26: Overlays of individual spectra of solutions containing 2 (blue), 2 and ${ }^{\text {tBuOH }}$ (orange) and only ${ }^{\text {BuOH (gray). }}$

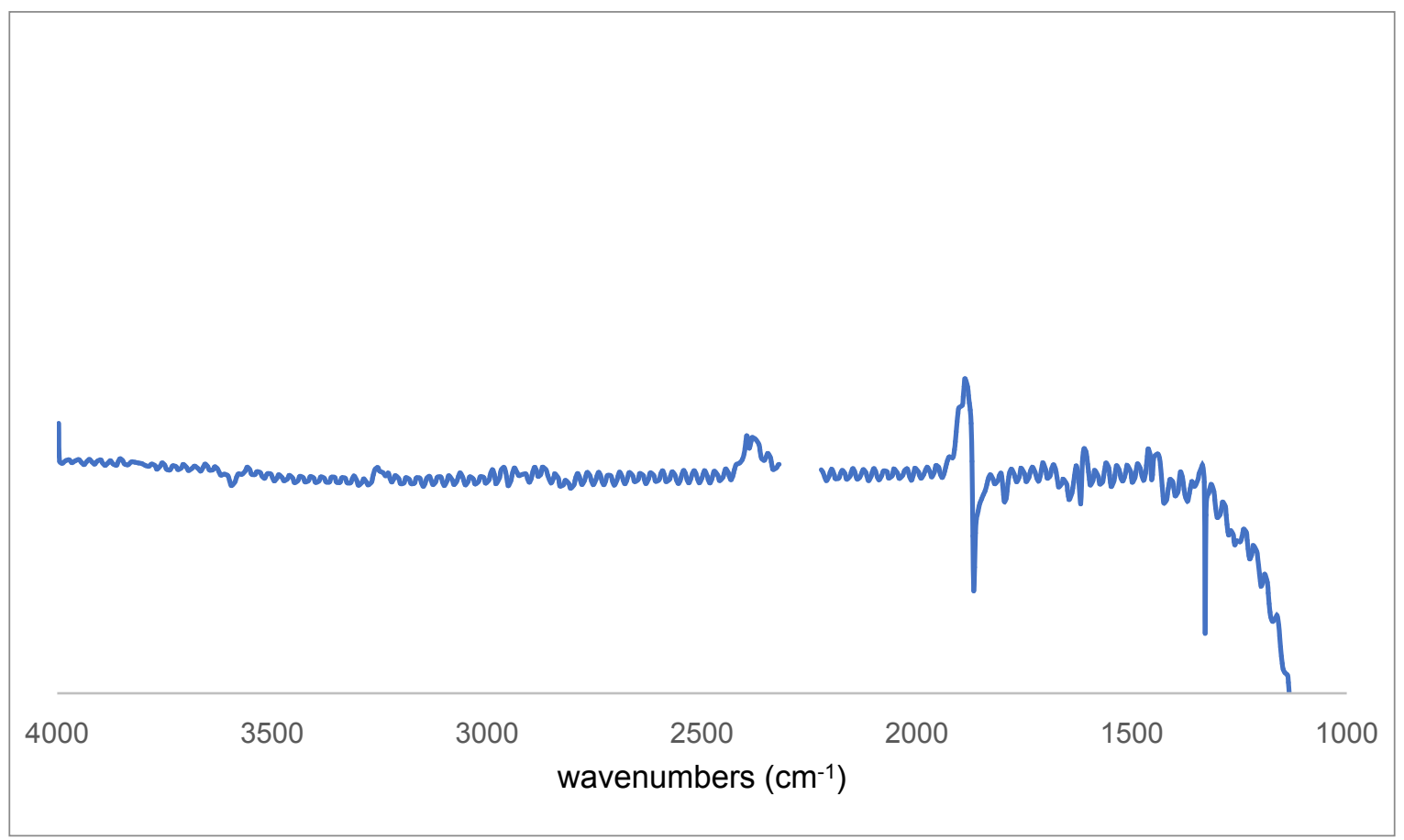

Figure S27: Difference spectrum determined by subtracting the spectra of 2 and only ${ }^{\text {tBuOH }}$ from the spectrum of the solution of 2 and ${ }^{t} \mathrm{BuOH}$. 


\section{SX. Empirical Rate Law Determinations for Conversion of $\mathbf{3}$ to 4 and 5 to 6}

In order to confirm that the reaction models used in numerical modelling were valid, the rate laws for the desired reactions were empirically determined. The rate laws for the conversion of $\mathbf{3}$ to $\mathbf{4}$ and 5 to $\mathbf{6}$ in the presence of $\mathrm{H}_{2}$ was determined by altering the initial concentrations of each starting material independently and determining the dependence of the observed rate constant, $k_{o b s}$, for those reaction sets. The rate dependence on partial pressure of $\mathrm{H}_{2}$ was not determined. The unshuttled and shuttled reactions conformed to the rate laws defined by Eq. 1 and Eq. 3, respectively (See Section SIII).

Rate dependence data for the reaction of 3 with $\mathrm{H}_{2}$ to form 4 :

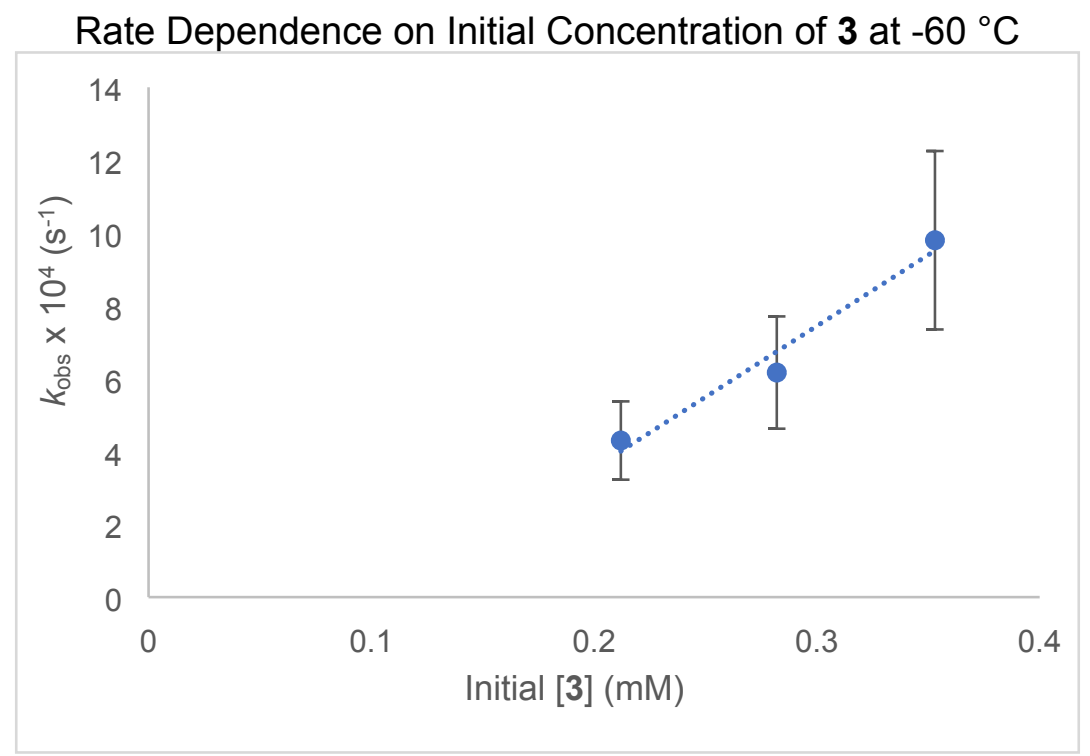

Figure S28: Dependence of $k_{\text {obs }}$ on initial concentration of $\mathbf{3}$ with 1 atm of $\mathrm{H}_{2}$ at $-60{ }^{\circ} \mathrm{C}$ as determined by the method of initial rates. 


\section{Rate Dependence on Initial Concentration of 3 at $-60{ }^{\circ} \mathrm{C}$ with ${ }^{\mathrm{t}} \mathrm{BuOH}$}

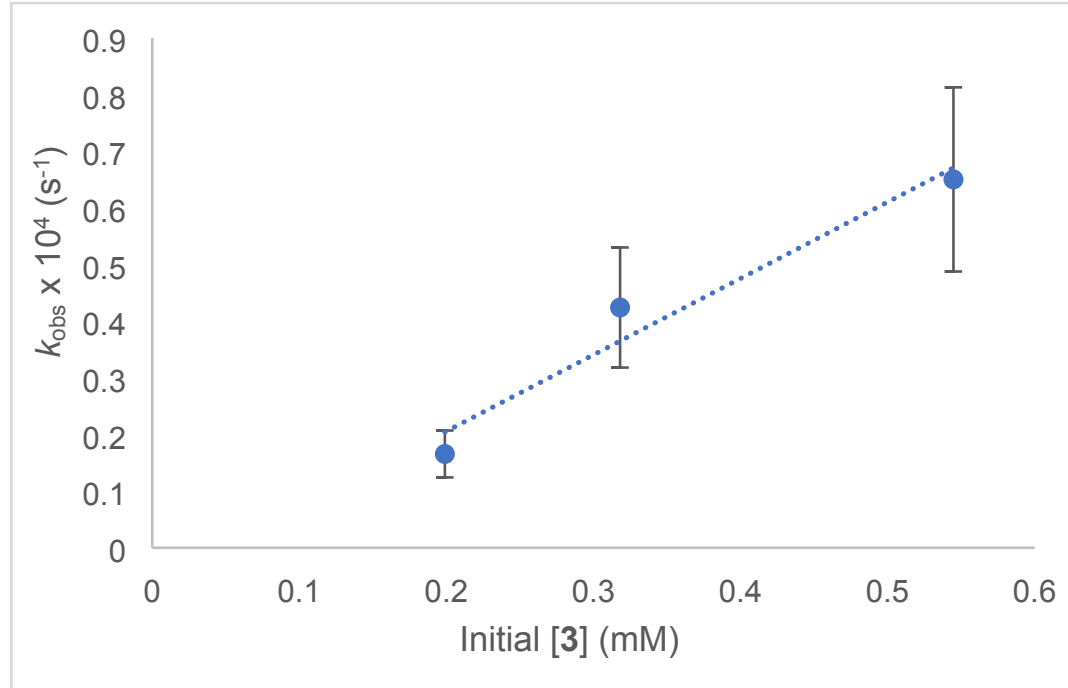

Figure S29: Dependence of $k_{\mathrm{obs}}$ on initial concentration of 3 with 1 atm of $\mathrm{H}_{2}$ at $-60{ }^{\circ} \mathrm{C}$ with $0.27 \mathrm{mM}$ t $\mathrm{BuOH}$ as determined by the method of initial rates.

Rate Dependence on Initial Concentration of ${ }^{\mathrm{t}} \mathrm{BuOH}$ at $-60^{\circ} \mathrm{C}$

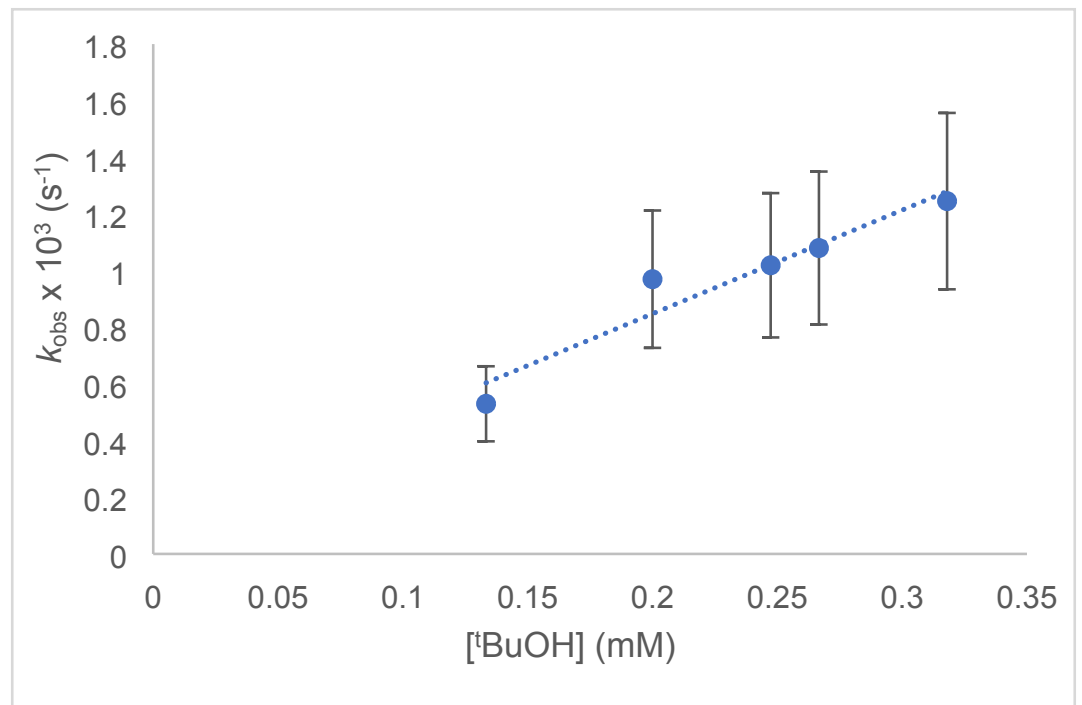

Figure S30: Dependence of $k_{\text {obs }}$ on initial concentration of ${ }^{\text {t }} \mathrm{BuOH}$ with $0.212 \mathrm{mM} 3$ and 1 atm of $\mathrm{H}_{2}$ at $-60{ }^{\circ} \mathrm{C}$ as determined by the method of initial rates. 


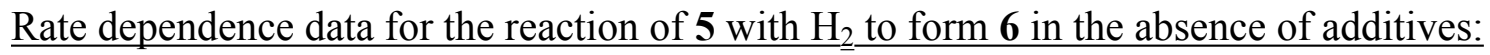

Rate Dependence on Initial Concentration of 5 at $25^{\circ} \mathrm{C}$

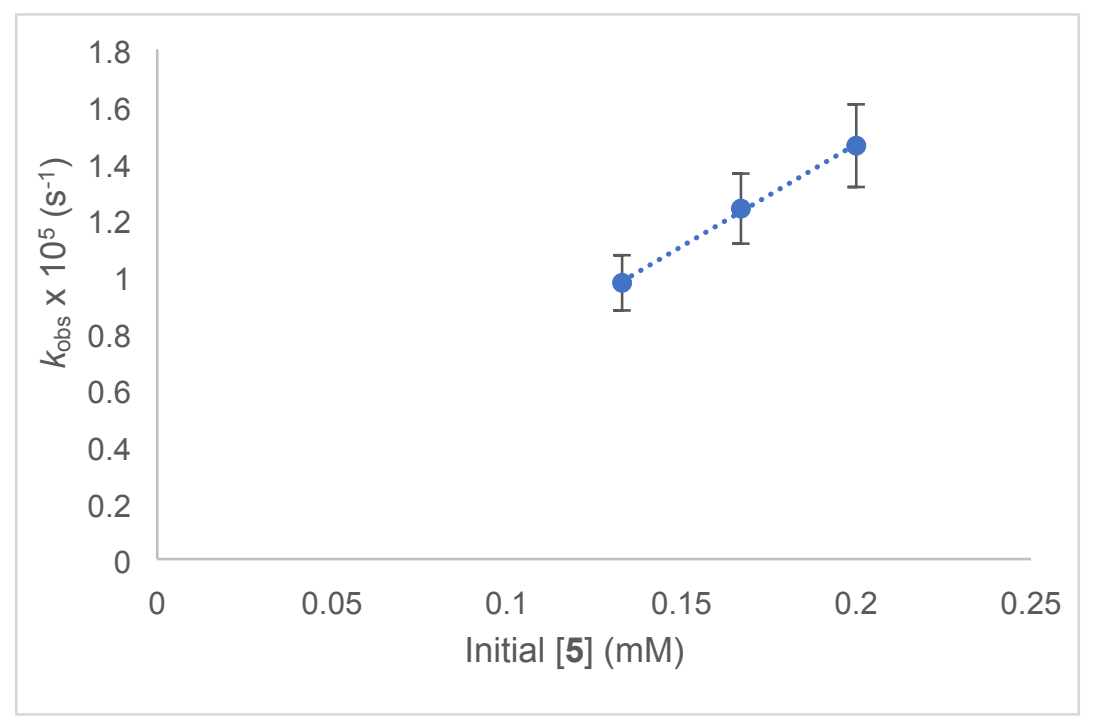

Figure S31: Dependence of $k_{\text {obs }}$ on initial concentration of 5 under 1 atm of $\mathrm{H}_{2}$ at $25{ }^{\circ} \mathrm{C}$ as determined by the method of initial rates.

Rate Dependence on Initial Concentration of ${ }^{\mathrm{B}} \mathrm{BuOH}$ at $25^{\circ} \mathrm{C}$

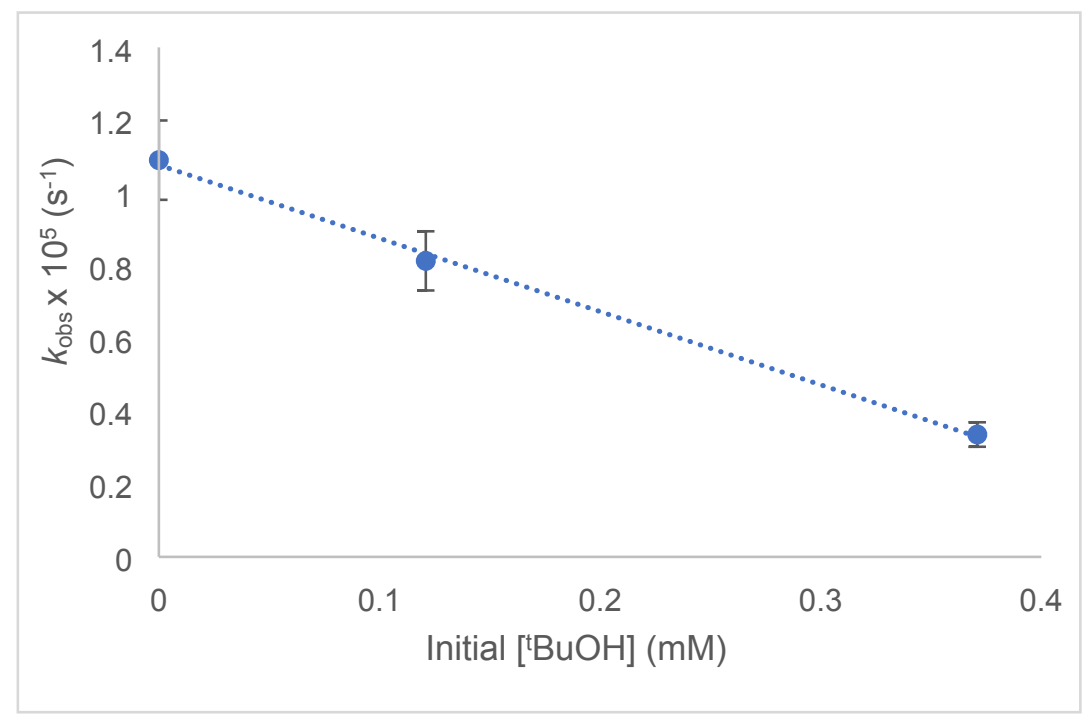

Figure S32: Dependence of $k_{\mathrm{obs}}$ on initial concentration of ${ }^{\mathrm{t}} \mathrm{BuOH}$ with $0.124 \mathrm{mM} 5$ and 1 atm of $\mathrm{H}_{2}$ at $25^{\circ} \mathrm{C}$ as determined by the method of initial rates. 
SXI. Information on Effect of Additives on $\mathrm{H}_{2}$ Activation by 3 and 5

Activation of $\mathrm{H}_{2}$ by both 3 and 5 can be enhanced by the addition of proton shuttles. Below are representative concentration profiles of the activation of $\mathrm{H}_{2}$ in the presence and absence of a proton shuttle. The amido complex 3 only requires 1 equiv. of $\mathrm{BuOH}$ in order for the rate of product formation to increase (Figure S30). Complex 5 requires an electron poor shuttle, $p$-bromo-2phenyl-2-propanol, in order to demonstrate the same effect (Figure S31). Variable-temperature ${ }^{1} \mathrm{H}$ NMR spectroscopy (Figure S32) of a solution containing 5 and one equiv. of ${ }^{\mathrm{t}} \mathrm{BuOH}$ suggests that there is an interaction between ${ }^{\mathrm{t}} \mathrm{BuOH}$ and $\mathbf{5}$. This is indicated by a shift in the hydride resonance of 5 from -25.85 to $-25.92 \mathrm{ppm}$, as well as shifting of the ligand backbone peaks, including the vinylic proton $\left(\mathrm{C}_{5} \mathrm{H}_{3} \mathrm{~N}\left(\mathrm{CHP}^{\mathrm{t}} \mathrm{Bu}_{2}\right)\right.$ that shifts from 3.49-3.62 ppm, both of which occur over a temperature range of 203 to $298 \mathrm{~K}$. We propose that this is consistent with transient binding of the alcohol to the open face of the 5-coordinate complex $\mathbf{5}$, as has been observed previously with a related complex. ${ }^{13}$

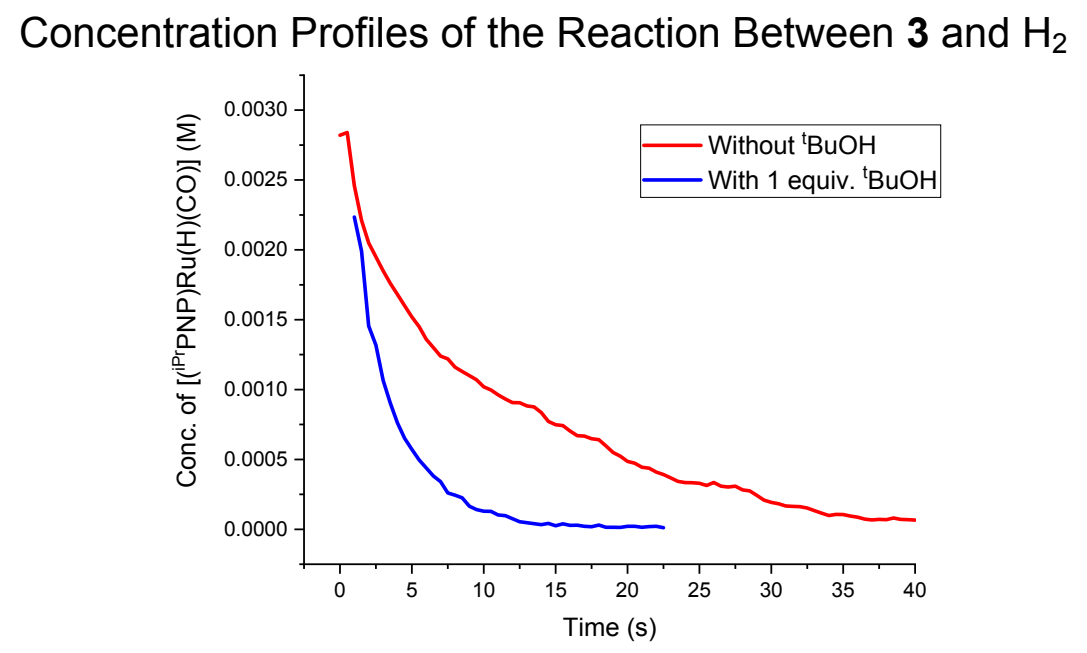

Figure S33: Line plots of the 1,2-addition of $\mathrm{H}_{2}$ to 3 in the presence (blue) and absence (red) of ${ }^{\mathrm{t}} \mathrm{BuOH}$. 
Concentration Profiles of the Reaction Between $\mathbf{5}$ and $\mathrm{H}_{2}$

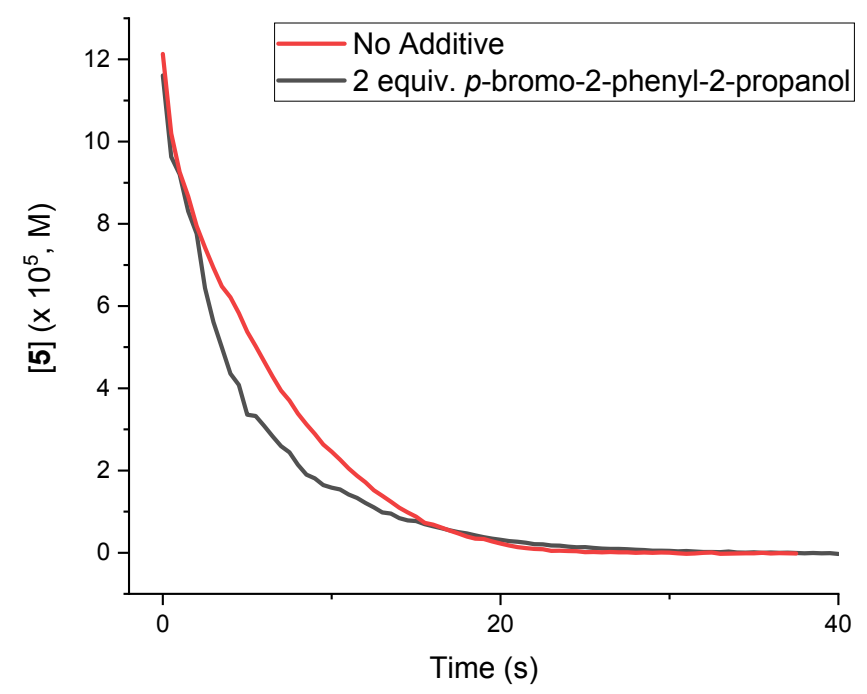

Figure S34: Line plots of the 1,2-addition of $\mathrm{H}_{2}$ to $\mathbf{5}$ in the presence (black) and absence (red) of $p$-bromo-2-phenyl-2-propanol.

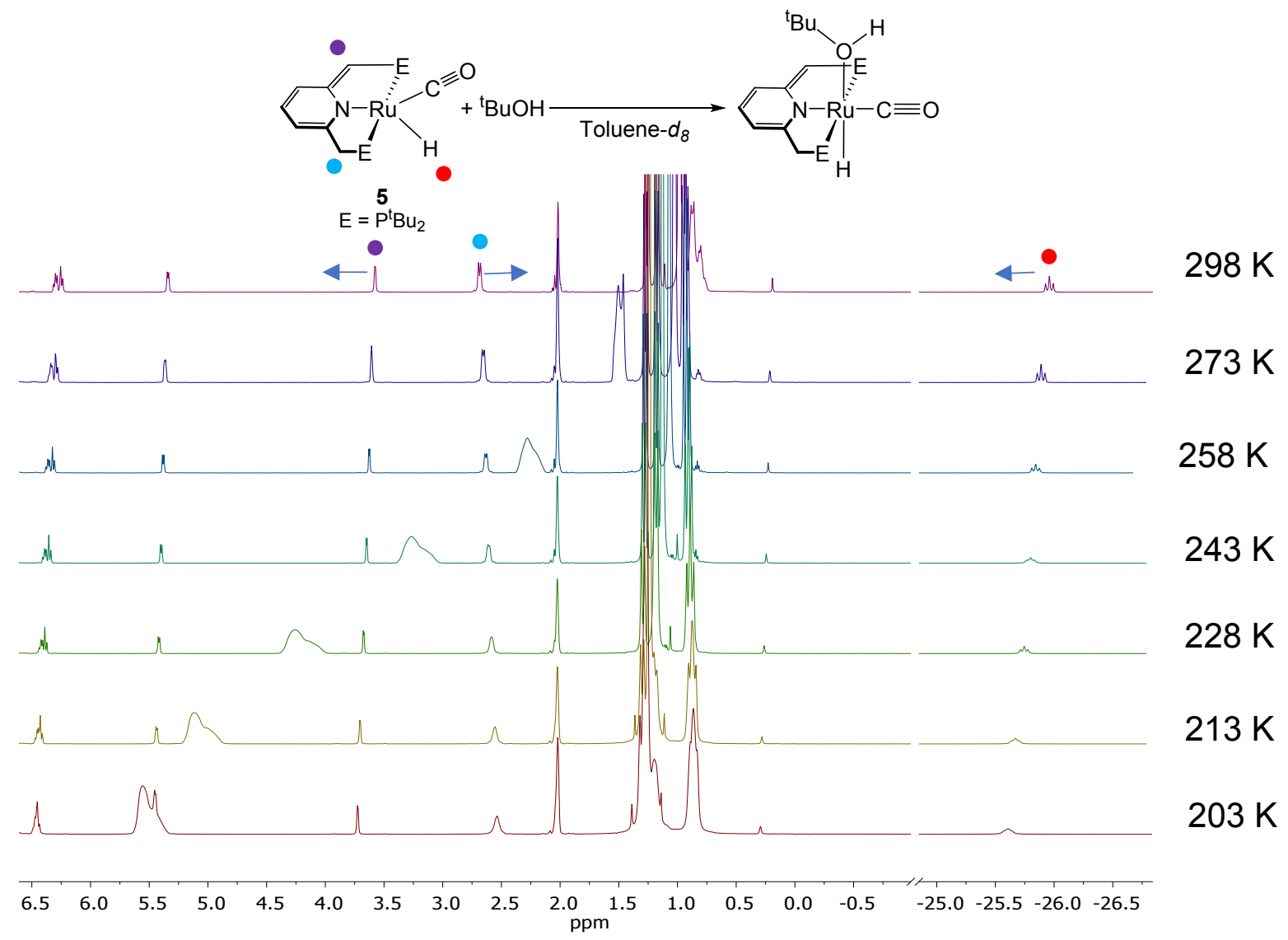

Figure S35: Variable temperature ${ }^{1} \mathrm{H}$ NMR data of 5 demonstrating broadening and shifting of ${ }^{\mathrm{t}} \mathrm{BuPN}{ }^{\mathrm{py}} \mathrm{P}^{*}$ and hydride resonances in the presence of 1 equiv. of ${ }^{\mathrm{t}} \mathrm{BuOH}$. Arrows show shift of peak as temperature decreases from $298 \mathrm{~K}$ to $203 \mathrm{~K}$. 


\section{SXII. References}

1. Koehne, I.; Schmeier, T. J.; Bielinski, E. A.; Pan, C. J.; Lagaditis, P. O.; Bernskoetter, W. H.; Takase, M. K.; Würtele, C.; Hazari, N.; Schneider, S. Synthesis and Structure of Six-Coordinate Iron Borohydride Complexes Supported by PNP Ligands. Inorg. Chem. 2014, 53, 2133-2143.

2. Bielinski, E. A.; Lagaditis, P. O.; Zhang, Y.; Mercado, B. Q.; Wurtele, C.; Bernskoetter, W. H.; Hazari, N.; Schneider, S. Lewis Acid-Assisted Formic Acid Dehydrogenation Using a PincerSupported Iron Catalyst. J. Am. Chem. Soc. 2014, 136, 10234-10237.

3. Nielsen, M.; Alberico, E.; Baumann, W.; Drexler, H.-J.; Junge, H.; Gladiali, S.; Beller, M. LowTemperature Aqueous-Phase Methanol Dehydrogenation to Hydrogen and Carbon Dioxide. Nature 2013, 495, 85.

4. (a) Li, Z.; Rayder, T. M.; Luo, L.; Byers, J. A.; Tsung, C.-K. Aperture-Opening Encapsulation of a Transition Metal Catalyst in a Metal-Organic Framework for $\mathrm{CO}_{2}$ Hydrogenation. J. Am. Chem. Soc. 2018, 140, 8082-8085; (b) Montag, M.; Zhang, J.; Milstein, D. Aldehyde Binding through Reversible $\mathrm{C}-\mathrm{C}$ Coupling with the Pincer Ligand upon Alcohol Dehydrogenation by a PNP-Ruthenium Catalyst. J. Am. Chem. Soc. 2012, 134, 10325-10328; (c) Zhang, L.; Nguyen, D. H.; Raffa, G.; Trivelli, X.; Capet, F.; Desset, S.; Paul, S.; Dumeignil, F.; Gauvin, R. M. Catalytic Conversion of Alcohols into Carboxylic Acid Salts in Water: Scope, Recycling, and Mechanistic Insights. ChemSusChem 2016, 9, 1413-1423.

5. Harris, R. K.; Becker, E. D.; Cabral De Menezes, S. M.; Granger, P.; Hoffman, R. E.; Zilm, K. W. Further Conventions for NMR Shielding and Chemical Shifts: (IUPAC recommendations 2008). Pure Appl. Chem. 2008, 80, 59-84.

6. (a) Bielinski, E. A.; Förster, M.; Zhang, Y.; Bernskoetter, W. H.; Hazari, N.; Holthausen, M. C. Base-Free Methanol Dehydrogenation Using a Pincer-Supported Iron Compound and Lewis Acid Co-catalyst. ACS Catal. 2015, 5, 2404-2415; (b) Lane, E. M.; Uttley, K. B.; Hazari, N.; Bernskoetter, W. Iron-Catalyzed Amide Formation from the Dehydrogenative Coupling of Alcohols and Secondary Amines. Organometallics 2017, 36, 2020-2025.

7. Shriver, D. F.; Drezdzon, M. A. The Manipulation of Air-Sensitive Compounds; Wiley: New Jersey, USA, 1986.

8. Nguyen, D. H.; Trivelli, X.; Capet, F.; Swesi, Y.; Favre-Réguillon, A.; Vanoye, L.; Dumeignil, F.; Gauvin, R. M. Deeper Mechanistic Insight into Ru Pincer-Mediated Acceptorless Dehydrogenative Coupling of Alcohols: Exchanges, Intermediates, and Deactivation Species. ACS Catal. 2018, 8, 4719-4734.

9. Bertoli, M.; Choualeb, A.; Lough, A. J.; Moore, B.; Spasyuk, D.; Gusev, D. G. Osmium and Ruthenium Catalysts for Dehydrogenation of Alcohols. Organometallics 2011, 30, 3479-3482.

10. Young, C. L. (Editor). Hydrogen and Deuterium; Pergamon Press: Elmsford, New York, 1981; Vol. 5.

11. Lee, C.; Xu, L.; Singhal, M.; Mendes, P.; Hoops, S.; Pahle, J.; Simus, N.; Gauges, R.; Sahle, S.; Kummer, U. COPASI - a COmplex PAthway SImulator. Bioinformatics 2006, 22, 3067-3074. 12. Liu, L.; Guo, Q.-X. Isokinetic Relationship, Isoequilibrium Relationship, and Enthalpy-Entropy Compensation. Chem. Rev. 2001, 101, 673-696.

13. Shubham, D.; Naziha, T.; Robert, F.; Sebastian, K.; Eugene, K. Unusual Rearrangement of Modified PNP Ligand based Ru Complexes Relevant to Alcohol Dehydrogenation Catalysis. Chem. Commun. 2019, 55, 11350-11353 